\title{
Die Erweiterung der umweltrechtsbehelfsfähigen Gegenstände auf dem Prüfstand*
}

\author{
Annette Guckelberger
}

\begin{abstract}
(C) Der/die Autor(en) 2020
Bei den umweltrechtsbehelfsfähigen Gegenständen folgt der UmwRG-Gesetzgeber weiterhin dem Enumerationsprinzip, während Art. 9 Abs. 3 Aarhus-Konvention generalklauselartig formuliert ist. Damit stellt sich die Frage der unions- und völkerrechtskonformen Ausgestaltung des deutschen Umweltrechtsschutzes. Der Beitrag beleuchtet, wie die Gerichte mit diesem schwierigen Problem umgehen. Außerdem wird aufgezeigt, dass angesichts unzureichender Umsetzung der Rechtsschutzvorgaben gegenüber Maßnahmen bzw. Unterlassungen der Organe und Einrichtungen der Union es wohl die nationalen Gerichte sind, die derartige Rechtsschutzlücken schließen müssten.
\end{abstract}

\section{Einführung}

Wie bereits im Beitrag im NuR, Heft 3/2020 dargelegt, ist die Erweiterung des Kreises der umweltrechtsbehelfsfähigen Gegenstände ein zentrales Anliegen der UmwRG-Novelle 2017. Auf diese Weise wollte man der Entscheidung V/9h der Vertragsstaatenkonferenz der Aarhus-Konvention $^{1}$ und der EuGH-Rechtsprechung zum unionsrechtlichen Umweltrecht i. V.m. Art. 9 Abs. 3 Aarhus-Konvention $^{2}$ (im Folgenden AK) Rechnung tragen und gleichsam der vom 7. Senat des BVerwG zur Behebung der Unzulänglichkeiten des deutschen Rechts entwickelten Figur eines prokuratorischen Rechts für eine Umweltvereinigung ${ }^{3}$ ein Ende setzen. Obwohl Art. 9 Abs. 3 AK generalklauselartig die Eröffnung des Zugangs von Mitgliedern der Öffentlichkeit zur Anfechtung von gegen umweltbezogene Rechtsvorschriften verstoßenden Handlungen bzw. Unterlassungen von Privatpersonen und Behörden vorsieht ${ }^{4}$ wird in $\$ 1$ Abs. 1 S. $1 \mathrm{UmwRG}^{5}$ festgelegt, dass dieses Gesetz nur auf Rechtsbehelfe gegen die dort genannten Entscheidungen anzuwenden ist. Mit der redaktionellen Ergänzung der Einleitung des $\$ 1$ Abs. 1 S. 1 UmwRG um das Wort „Entscheidung“ wollte man einen Oberbegriff einführen, um klarzustellen, dass es verschiedene Entscheidungsarten gibt, die angesichts des Bezugs auf das Fachrecht bei den einzelnen Kategorien des Katalogs z. B. Genehmigungen oder Planfeststellungsbeschlüsse umfassen. ${ }^{6}$

Damit folgt das deutsche Recht dem Listenprinzip. ${ }^{7}$ Dieses Vorgehen lässt sich damit erklären, dass hinsichtlich der Rechtsbehelfe von Vereinigungen gegen die jeweiligen Entscheidungen je nach Kategorie unterschiedliche Maßgaben gelten. Da Art. 9 Abs. 3 AK im Unterschied zu Absatz 2 den Gerichtszugang lediglich bei Verstößen gegen umweltbezogene Bestimmungen verlangt, muss eine Vereinigung gem. $\$ 2$ Abs. 1 S. 2 UmwRG bei Rechtsbehelfen gegen Entscheidungen nach $\$ 1$ Abs. 1 S. 1 Nrn. $2 a-6$ UmwRG bei der Rügebefugnis zudem die Verletzung umweltbezogener Rechtsvorschriften geltend machen und sind derartige Rechtsbehelfe nach $\$ 2$ Abs. 4 S. 1 Nr. 2 UmwRG nur begründet, wenn die Entscheidung oder de-

Prof. Dr. Annette Guckelberger,

Lehrstuhl für Öffentliches Recht, Universität des Saarlandes, Saarbrücken, Deutschland ren Unterlassung gegen umweltbezogene Rechtsvorschriften verstößt. ${ }^{8}$ Ferner wird gem. $\$ 7$ Abs. 4 UmwRG nur in Rechtsbehelfsverfahren gegen Entscheidungen nach $\$ 1$ Abs. 1 S. 1 Nrn. 1-2b UmwRG die materielle Präklusion aus $\$ 73$ Abs. 4 S. 3-6 VwVfG für unanwendbar erklärt, während die einer Pflicht zur Strategischen Umweltverträglichkeitsprüfung (SUP) unterliegenden Pläne und Programme i.S.d. $\$ 1$ Abs. 1 S. 1 Nr. 4 UmwRG - abgesehen von Bauleitplänen nach $\int 7$ Abs. 3 UmwRG - einer materiellen Präklusion unterliegen. Zwar ist das Listenprinzip nicht per se völker- bzw. unionsrechtswidrig, sofern der gesetzliche Katalog alle vom Völker- und Unionsrecht erfassten Bereiche abdeckt. ${ }^{9}$ Schlacke ist jedoch darin beizupflichten, dass angesichts der dynamischen Haltung des Aarhus Compliance Committee (ACC) sowie des EuGH das Listenprinzip zur Implementierung damit in Zusammenhang stehender Neuerungen zu unflexibel ist. ${ }^{10}$

Während die in \$1 Abs. 1 S. 1 Nrn. 1-3 UmwRG genannten Entscheidungen bereits nach früherem Recht den Rechtsbehelfen des UmwRG unterfielen, wurde der bestehende Katalog im Hinblick auf Art. 9 Abs. 3 AK um die Nrn. 4-6 erweitert und dadurch die Anpassung des deutschen Rechts um einen großen Schritt vorangetrieben. ${ }^{11} \mathrm{Da}$

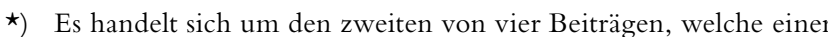
Langfassung ihres Vortrag in Berlin am 2.12.2019 auf dem „Forum Umweltrechtsschutz 2019: Erfahrungen mit der Novelle des Umwelt-Rechtsbehelfsgesetzes (UmwRG) seit 2017“, veranstaltet durch das Unabhängige Institut für Umweltfragen, entsprechen.

1) Aarhus Vertragsstaatenkonferenz, Entscheidung V/9h betreffend die Beschwerde über Deutschland, ECE/MP.PP/2014/2/Add.1, S. 66f, Stand 16.12.2019, abrufbar unter http://www.unece.org/ fileadmin/DAM/env/pp/mop5/Documents/Post_session_docs/ ece_mp.pp_2014_2_add.1_eng.pdf.

2) Übereinkommen über den Zugang zu Informationen, die Öffentlichkeitsbeteiligung an Entscheidungsverfahren und den $\mathrm{Zu}$ gang zu Gerichten in Umweltangelegenheiten v. 25.6.1998.

3) BVerwG, Urt. vom 5.9.2013 - 7 C 21.12, BVerwGE 147, 312 , 324f; s. auch BT-Drs. 18/9526, S. 23 f.; Buchberger, EurUP 2019, 377, $380 \mathrm{f}$.

4) S. Heß, ZUR 2018, 686, 688, wonach aus der Gegenüberstellung von Art. 9 Abs. 2 und Abs. 3 AK sich bei Letzterem gerade keine Beschränkung der angreifbaren Handlungen ergibt.

5) Gesetz über ergänzende Vorschriften zu Rechtsbehelfen in Umweltangelegenheiten nach der EG-Richtlinie 2003/35/ EG (Umwelt-Rechtsbehelfsgesetz - UmwRG) v. 7.12.2006, BGBl. I S. 2816; zuletzt geändert durch Artikel 4 des Gesetzes v. 17. 12.2018, BGB1. I S. 2549.

6) BT-Drs. 18/9526, S. 31

7) Schlacke, EurUP 2018, 127, 140

8) $\mathrm{Zu}$ dieser Ursache für die Unterscheidung auch Franzius, in: Schink/Reidt/Mitschang, UVPG/UmwRG, 2018, \1 UmwRG Rdnr. 9f.

9) Schlacke, EurUP 2018, 127, 140.

10) Schlacke, EurUP 2018, 127, 140; s. auch Franzius, in: Schink/ Reidt/Mitschang, UVPG/UmwRG, 2018, \$1 UmwRG Rdnr. 3; Schieferdecker, in: Hoppe/Beckmann/Kment, UVPG/ UmwRG, 5. Aufl. 2018, \$1 UmwRG Rdnr. 24.

11) So Schlacke, EurUP 2018, 127, 140. 
sich in diesem Kontext Abgrenzungsfragen zu $₫ 1$ Abs. 1 S. 1 Nr. 1 UmwRG ergeben können, wird zugleich überblicksartig auf diese schon vor 2017 bestehende Regelung eingegangen.

\section{2. $\$ 1$ Abs. 1 S. 1 Nr. 1 UmwRG}

Gem. $₫ 1$ Abs. 1 S. 1 Nr. 1 UmwRG findet dieses Gesetz auf Rechtsbehelfe gegen Zulassungsentscheidungen i.S.v. $\$ 2$ Abs. $6 \mathrm{UVPG}^{12}$ über die Zulässigkeit von Vorhaben Anwendung, für die nach lit. a) dem Gesetz über die Umweltverträglichkeitsprüfung, lit. b) der Verordnung über die Umweltverträglichkeitsprüfung bergbaulicher Vorhaben oder lit. c) landesrechtlichen Vorschriften eine Pflicht zur Durchführung einer UVP bestehen kann. Während die überwiegende Meinung zu Recht unter Rekurs auf die Verwendung des Verbs ,kann“ für die Zulässigkeit des Umweltrechtsbehelfs die Möglichkeit einer UVP für das Vorhaben genügen lässt, ${ }^{13}$ ist nach Ansicht des BVerwG das Bestehen der UVP-Pflicht bereits im Rahmen der Zulässigkeitsprüfung abschließend zu klären. ${ }^{14}$ Damit entfernt es sich nicht nur vom Gesetzeswortlaut, sondern auch von den Gesetzesmaterialien, denen zufolge bei Vorprüfungen des Einzelfalls ,,im Rahmen der Begründetheit des Rechtsbehelfs also primär zu prüfen [ist], ob eine UVP durchgeführt wurde bzw. hätte durchgeführt werden müssen". ${ }^{15} \mathrm{Da}$ im Zuge der UmwRG-Novelle 2017 dieselben Erwägungen auf die neu eingefügte Nr. 4 übertragen wurden, ${ }^{16}$ sind diese Aussagen auch nicht als obsolet anzusehen.

$\mathrm{Zu}$ den Nr. 1 unterfallenden Zulassungsentscheidungen gehören z.B. Planfeststellungsbeschlüsse für den Bau einer Bundesautobahn (\$.6 UVPG i. V.m. Anlage 1 Ziff. 14.3 zum UVPG), ${ }^{17}$ ein Änderungsplanfeststellungsbeschluss für ein Deponievorhaben mit einer Gesamtkapazität von 2599200 Tonnen ( $\$ 35$ Abs. 2 KrWG, \$6 UVPG i.V.m. Anlage 1 Ziff. 12.2.1 zum UVPG), ${ }^{18}$ Waldumwandlungsgenehmigungen bei einer Fläche von mehr als 10 Hektar (Anlage 1 Ziff. 17.2.1 zum UVPG), ${ }^{19}$ die Zulassung von Windfarmen mit Anlagen einer Gesamthöhe von jeweils mehr als 50 Meter nach Maßgabe der Anlage 1 Ziff. 1.6 zum $\mathrm{UVPG}^{20}$ und Zulassungsentscheidungen zur Intensivhaltung oder -aufzucht von Mastgeflügel nach Maßgabe der Anlage 1 Ziff. 7.3 zum UVPG. ${ }^{21}$

Zulassungsentscheidungen werden in $\$ 2$ Abs. $6 \mathrm{Nr}$. 1 UVPG - mit Ausnahme der Anzeigeverfahren - als die Bewilligung, Erlaubnis, Genehmigung, der Planfeststellungsbeschluss und sonstige behördliche Entscheidungen über die Zulässigkeit von Vorhaben, die in einem Verwaltungsverfahren getroffen werden, einschließlich des Vorbescheids umschrieben. Nach der EuGH-Rechtsprechung ist der Begriff der Genehmigung in Art. 1 Abs. 2 lit. c) UVPRL 2011/92/EU ${ }^{22}$ weit zu verstehen. ${ }^{23}$ Daher müssen diese nicht notwendig in eine Entscheidung i.S.d. $\$ 9 \mathrm{VwVfG}$ (= Verwaltungsakte oder öffentlich-rechtliche Verträge) münden, vielmehr können darunter auch Zulassungsentscheidungen politischer Gremien fallen, wie zum Beispiel der Beschluss des Rates der Stadt Essen zum Bau und Baubeginn der Maßnahme „Verkehrskonzept Werden“" (unter Verweis auf Ziff. 14.6 Anlage 1 zum UVPG). ${ }^{24}$ Im August 2019 entschied der VGH Kassel, dass die Entscheidung über die Zielabweichung von Raumordnungsplänen (\$ 6 Abs. 2 ROG) in Bezug auf ein an und für sich UVP-pflichtiges Logistikzentrum nicht unter $\int 1$ Abs. 1 S. 1 Nr. 1 UmwRG i. V.m. $\$ 2$ Abs. 6 Nr. 1 UVPG subsumiert werden könne, weil durch diese Entscheidung das Logistikzentrum als Vorhaben nicht zugelassen werde. Zwar sei das Zielabweichungsverfahren conditio sine qua non für die spätere $\mathrm{Zu}$ lassung des Vorhabens, aber nicht in diesem, sondern erst später werde über die Zulässigkeit des konkreten Vorhabens entschieden. Für die in $\$ 2$ Abs. 6 Nr. 1 UVPG erwähnten Ausnahmen und Befreiungen sei charakteristisch, dass diese neben die Zulassungsentscheidung treten. Bei der
Zielabweichung werde jedoch nur vom Anpassungsgebot des $₫ 1$ Abs. 4 BauGB befreit. Als weiteres Argument wird angeführt, dass auch Raumordnungspläne mangels Entscheidung über die Zulassung konkreter Vorhaben nicht $\$ 1$ Abs. 1 S. 1 Nr. 1 UmwRG unterfielen. ${ }^{25}$

$\mathrm{Zu}$ beachten ist aber, dass nach der Legaldefinition in $\$ 2$ Abs. 6 Nr. 3 UVPG auch Beschlüsse über die Aufstellung, Änderung oder Ergänzung von Bebauungsplänen, durch welche die Zulässigkeit von bestimmten Vorhaben i.S.d. Anlage 1 begründet wird, ${ }^{26}$ sowie Beschlüsse über Bebauungspläne, die Planfeststellungsbeschlüsse i.S.d. Anlage 1 ersetzen, ebenfalls $₫ 1$ Abs. 1 S. 1 Nr. 1 UmwRG unterfallen. Als Beispiele dafür seien ein vorhabenbezogener Bebauungsplan etwa für die Errichtung einer Tierhaltungsanlage mit 3168 Zuchtsauen ( $\$ 2$ Abs. 6 Nr. 3 UVPG i.V.m. Anlage 1 Ziff. 7.8.1 zum UVPG ${ }^{27}$ oder einer Windfarm gem. $\ 2$ Abs. 6 Nr. 3 UVPG i. V.m. Anlage 1 Ziff. 1.6 zum UVPG genannt. ${ }^{28}$

12) Gesetz über die Umweltverträglichkeitsprüfung (UVPG) v. 24. 2.2010, BGBl. I S. 94; zuletzt geändert durch Art. 22 des Gesetzes v. 13.5.2019, BGBl. I S. 706.

13) OVG Lüneburg, Urt. v. 27.8.2019 - 7 KS 24/17, Rdnr. 100, juris; VGH Mannheim, Urt. v. 20.11.2018 - 5 S 2138/16, Rdnr. 73, juris; VG Hannover, Beschl. v. 28.3.2019 - 4 B 5526/18, Rdnr. 63, juris; Bunge, in: ders., UmwRG, 2. Aufl. 2019, \$1 Rdnr. 53; Fellenberg/Schiller, in: Landmann/Rohmer, Umweltrecht, 90. EL Juni 2019, UmwRG \$1 Rdnr. 39f.; Franzius, in: Schink/Reidt/Mitschang, UVPG/UmwRG, 2018, \$1 UmwRG Rdnr. 17; Schlacke, in: Gärditz, VwGO, 2. Aufl. 2018, $\$ 1$ UmwRG Rdnr. 23; s. auch Schieferdecker, in: Hoppe/Beckmann/Kment, UVPG/UmwRG, 5. Aufl. 2018, \$1 UmwRG Rdnr. 40, wonach objektiv die Pflicht zur Vorprüfung bestehen muss, im Übrigen aber die Möglichkeit genügt, dass die Vorprüfung zu einer UVP-Pflicht führt.

14) BVerwG, Urt. v. 2.11.2017 - 7 C 25.15, NVwZ 2018, 986; Happ, in: Eyermann/Fröhler, VwGO, 15. Aufl. 2019, $\$ 1$ UmwRG Rdnr. 10.

15) BT-Drs. 16/2495, S. 11, Kursivhervorhebung durch die Verfasserin.

16) BT-Drs. 18/9526, S. 33.

17) Zur früheren Rechtslage OVG Münster, Urt. v. 11.9.2019 11 D 81/16.AK, Rdnr. 102, juris.

18) OVG Lüneburg, Urt. v. 31.7.2018 - 7 KS 17/16, Rdnr. 85, juris.

19) VG Freiburg, Beschl. v. 15.2.2019 - 10 K 536/19, NuR 2019, $356,357$.

20) Z.B. VG Wiesbaden, Beschl. v. 22.8.2019 - 4 L 2007/18, WI Rdnr. 33 ff., juris zum früheren UVPG.

21) Z.B. VG Hannover, Beschl. v. 28.3.2019 - 4 B 5526/18, Rdnr. 62f., juris; VG München, Urt. v. 22.3.2019 - M 19 K 17.3738, AUR 2019, 346, 348.

22) Richtlinie 2011/92/EU des Europäischen Parlaments und des Rates vom 13.12.11 über die Umweltverträglichkeitsprüfung bei bestimmten öffentlichen und privaten Projekten, ABl. 1985 L 175, S. 40 ff. (zuletzt geändert durch Richtlinie 2014/52/EU des Europäischen Parlaments und des Rates vom 16.4.2014 zur Änderung der Richtlinie 2011/92/EU über die Umweltverträglichkeitsprüfung bei bestimmten öffentlichen und privaten Projekten, AB1. 2014 L 124, S. 1).

23) S. EuGH, Urt. v. 4. 5.2006 - C-290/03, Slg 2006, I-3949-3968, Rdnr. $44 \mathrm{ff}$.

24) OVG Münster, Beschl. v. 11. 4.2018 - 8 B 1463/17, NVwZ-RR 2018, 642, 644f.

25) VGH Kassel, Beschl. v. 15.8.2019 - 4 B 1303/19, Rdnr. 33 ff.; nicht zum Abweichungsverfahren, aber in diese Richtung Franzius, in: Schink/Reidt/Mitschang, UVPG/UmwRG, 2018, $\$ 1$ UmwRG Rdnr. 15.

26) Näher dazu Happ, in: Eyermann/Fröhler, VwGO, 15. Aufl 2019, \$1 UmwRG Rdnr. 8; s. auch Bunge, in: ders., UmwRG, 2. Aufl. 2019, $\$ 1$ Rdnr. 62 ff., wonach die Beschränkung auf vorhabenbezogene Bebauungspläne sich nur schwer mit den Entwicklungen im Bauplanungsrecht vereinbaren lasse.

27) OVG Lüneburg, Urt. v. 11.12.2018 - 1 KN 185/16, UPR 2019, 264,266

28) OVG Lüneburg, Urt. v. 27.9.2018 - 12 KN 191/17, NuR 2018, 780. 


\section{3. $\$ 1$ Abs. 1 S. 1 Nr. 4 UmwRG}

Seit der UmwRG-Novelle 2017 können sich Umweltrechtsbehelfe gem. $\$ 1$ Abs. 1 Nr. 4 HS 1 UmwRG gegen Entscheidungen über die Annahme von Plänen und Programmen i.S.d. $\$ 2$ Abs. $7 \mathrm{UVPG}^{29}$ und entsprechender landesrechtlicher Vorschriften richten, für die nach a) Anlage 5 des UVPG oder b) landesrechtlichen Vorschriften eine Pflicht zur Durchführung einer SUP bestehen kann. Diese kann sich entweder daraus ergeben, dass der konkrete Plan oder das konkrete Programm nach den gesetzlichen Vorgaben zwingend einer SUP bedarf, oder aus dem Ergebnis einer Vorprüfung des Einzelfalls. ${ }^{30} \mathrm{Wie}$ bereits erwähnt, ist dieser Frage nach den Gesetzesmaterialien primär im Rahmen der Begründetheit nachzugehen. ${ }^{31}$

\subsection{SUP-pflichtige Pläne und Programme}

Gem. $₫ 35$ Abs. 1 UVPG ist eine SUP bei Plänen und Programmen durchzuführen, wenn sie in Anlage $5 \mathrm{Nr}$. 1 aufgeführt sind (Nr. 1), wie dies z.B. bei Risikomanagementplänen nach \$75 WHG, Maßnahmenprogrammen nach \82 WHG, Raumordnungsplänen nach \13 ROG sowie bei Bauleitplanungen nach $\$ \$ 6,10$ BauGB der Fall ist. Eine SUP ist aber auch bei Plänen und Programmen durchzuführen, die in Nr. 2 besagter Anlage aufgeführt sind (z.B. Lärmaktionspläne nach $\$ 47 \mathrm{~d}$ BImSchG, Luftreinhaltepläne nach $\int 47$ Abs. 1 BImSchG, Abfallwirtschaftskonzepte nach $\$ 21 \mathrm{KrWG}$ ) und für Entscheidungen über die Zulässigkeit von in der Anlage 1 aufgeführten oder von solchen Vorhaben, die nach Landesrecht einer SUP oder einer Vorprüfung des Einzelfalls bedürfen, einen Rahmen setzen ( $\$ 35$ Abs. 1 Nr. 2 UVPG). Letzteres ist gem. $\$ 35$ Abs. 3 UVPG der Fall, wenn sie Festlegungen mit Bedeutung für spätere Zulassungsentscheidungen enthalten, insbesondere $\mathrm{zu} B e-$ darf, Standort, Beschaffenheit, Betriebsbedingungen von Vorhaben oder Inanspruchnahme von Ressourcen. Laut den Materialien muss der Plan keine zwingenden Vorgaben für spätere Vorhaben treffen, ausreichend ist, wenn diese bei Abwägungsentscheidungen, bei der Ausübung von Ermessen oder im Rahmen von Beurteilungsermächtigungen $\mathrm{zu}$ berücksichtigen sind. ${ }^{32}$ Ist dies der Fall, ist es unerheblich, dass dem Plan keine unmittelbare rechtliche Außenwirkung zukommt. ${ }^{33}$

Die Neuregelung bezieht sich unter anderem auf Flächennutzungs- und Bebauungspläne, die SUP-pflichtig sind $^{34}$ (nicht aber auf Bebauungspläne nach $\$ \int 13,13 \mathrm{a}, 13 \mathrm{~b}$ BauGB $\left.^{35}\right)$. Da nach dem Gesetzeswortlaut des $₫ 1$ Abs. 1 S. 1 Nr. 4 UmwRG die Entscheidung die „Annahme“ der Pläne betreffen muss, kann - nach nicht unumstrittener Meinung - darunter nicht die Genehmigung des Flächennutzungsplans nach $₫ 6$ BauGB $^{36}$ oder des Bebauungsplans nach $\$ 10$ Abs. 2 BauGB subsumiert werden. Vielmehr ist insoweit auf $\int 1$ Abs. 1 S. 1 Nr. 6 UmwRG zu rekurrieren. ${ }^{37}$ Auch in der Ânderung derartiger Pläne kann man eine Entscheidung über deren Annahme sehen, wohingegen deren Aufhebung oder die Einstellung eines Aufstellungsverfahrens eher der über $\$ 1$ Abs. $1 \mathrm{~S}$. 2 UmwRG ebenfalls erfassten Untätigkeit zuzuordnen sind. ${ }^{38}$

Obwohl man nach den Materialien mit $₫ 1$ Abs. 1 S. 1 Nr. 4 UmwRG der BVerwG-Rechtsprechung zur umweltrechtlichen Verbandsklage in Bezug auf Luftreinhaltepläne Rechnung tragen wollte, ${ }^{39}$ stellte sich für die Rechtsprechung die Frage, wie mit dieser Neuerung umzugehen ist, insbesondere ob Umweltrechtsbehelfe bereits unzulässig sind, wenn derartige Pläne tatsächlich nicht SUP-pflichtig sind. Da Luftreinhaltepläne im Anhang 5 zum UVPG unter Ziff. 2.2 aufgeführt sind, unterfallen sie gem. $\$ 35$ Abs. 1 Nr. 2 UVPG einer konditionalen Pflicht zur SUP, wenn sie rahmensetzend sind. ${ }^{40}$ Das OVG Münster hält eine Rahmensetzung durch einen solchen Plan grundsätzlich für möglich, sofern er Maßnahmen zur dauerhaften Vermin- derung von Luftverunreinigungen enthält, die für die Genehmigung UVP-pflichtiger Vorhaben relevant werden können, etwa in Bezug auf die in der Anlage 1 zum UVPG aufgezählten Verkehrsvorhaben, wie Ziff. 14.11 (Bau von Straßenbahn- oder U-Bahnstrecken). ${ }^{41}$ Unter Rekurs auf die Materialien und den Gesetzeswortlaut („kann“) betonen die Gerichte, dass es für die Zulässigkeit nur auf die Möglichkeit einer SUP-Pflicht ankomme. ${ }^{42}$ Bei einem Unterlassen oder Fortschreiben eines Luftreinhalteplans sei eine solche $\mathrm{zu}$ bejahen, wenn diese nicht offensichtlich ausgeschlossen ist. ${ }^{43}$ Da nach $\$ 7$ Abs. 2 S. 1 UmwRG Rechtsbehelfe gegen das Unterlassen von Entscheidungen nach \1 Abs. 1 S. 1 Nr. 4 UmwRG möglich sein sollen, sprächen praktische Gesichtspunkte gegen eine enge Normauslegung, denn andernfalls werde ein beträchtlicher Teil des materiellen Prüfprogramms in die Zulässigkeitsprüfung verlagert. ${ }^{44}$ Zudem lege das Unionsrecht einen möglichst effektiven Rechtsschutz für Verbände nahe. ${ }^{45}$ Ansonsten hinge - so das VG Hannover - die Klagebefugnis allein davon ab, ob die Beklagte bzw. Antragsgegnerin in ihrem Luftreinhalteplan die Festschreibung von Maßnahmen intendiert, die für Vorhaben nach der Anlage 1 zum UVPG rahmensetzend sind. ${ }^{46}$ Die Rechtsprechung des BVerwG, dass bei $\$ 1$ Abs. $1 \mathrm{~S}$. 1 Nr. 1 UmwRG bereits im Rahmen der Zulässigkeit des Rechtsbehelfs das tatsächliche Bestehen der UVP-Pflicht des Vorhabens festzustellen ist, sei auf $\$ 1$ Abs. 1 S. 1 Nr. 4 UmwRG nicht übertragbar, weil sich die Nr. 1 auf bereits erlassene Zulassungsentscheidungen mit bekanntem und auf eine UVP-Pflicht überprüfbarem Inhalt beziehe. ${ }^{47}$

Sollte $\$ 1$ Abs. 1 S. 1 Nr. 4 UmwRG aufgrund engerer Auslegung nicht anwendbar sein, ist nach dem OVG Münster diese Vorschrift jedenfalls angesichts einer plan-

29) Dazu, dass manchen die dortige Legaldefinition zu eng ist, und zur Notwendigkeit einer ggf. unionsrechtskonformen Auslegung Fellenberg/Schiller, in: Landmann/Rohmer, Umweltrecht, 90. EL 6/2019, UmwRG \$ 1 Rdnr. $75 \mathrm{ff}$.

30) BT-Drs. 18/9526, S. 33.

31) BT-Drs. 18/9526, S. 33.

32) BT-Drs. 18/9526, S. 34.

33) BT-Drs. 18/9526, S. 34

34) BT-Drs. 18/9526, S. 34.

35) Schlacke, NVwZ 2019, 1392, 1398

36) OVG Koblenz, Beschl. v. 4. 7.2018 - 8 E 10238/18, Rdnr. 13, juris.

37) OVG Koblenz, Beschl. v. 4.7.2018 - 8 E 10238/18, Rdnr. 13, juris; Fellenberg/Schiller, in: Landmann/Rohmer, Umweltrecht, 90. EL Juni 2019, UmwRG $\$ 1$ Rdnr. 22; a. A. Schieferdecker, in: Hoppe/Beckmann/Kment, UVPG/UmwRG, 5. Aufl. 2018, \$1 UmwRG, Rdnr. 61.

38) Schieferdecker, in: Hoppe/Beckmann/Kment, UVPG/UmwRG, 5. Aufl. 2018, \1 UmwRG, Rdnr. 62.

39) BT-Drs. 18/9526, S. 35

40) OVG Münster, Beschl. v. 6.12.2018 - 8 D 62/18.AK, ZUR 2019, 97, 98; s. auch VGH Kassel, Beschl. v. 17.12.2018 - 9 A 2037/18.Z, NVwZ 2019, 329, 331.

41) OVG Münster, Beschl. v. 6.12.2018 - 8 D 62/18.AK, ZUR 2019, 97, 98

42) OVG Münster, Beschl. v. 6.12.2018 - 8 D 62/18.AK, ZUR 2019, 97, 99; VGH Kassel, Beschl. v. 17. 12.2018 - 9 A 2037/18.Z, NVwZ 2019, 329, 331; VG Hannover, Beschl. v. 25. 10.2018 4 A 11790/17, Rdnr. 10, 13, juris; VG Sigmaringen, Beschl. v. 21. 8. 2018 - 1 K 1825/18, ZUR 2018, 631, 632.

43) OVG Münster, Beschl. v. 6.12.2018 - 8 D 62/18.AK, ZUR 2019, 97, 99.

44) VG Hannover, Beschl. v. 25.10.2018 - 4 A 11790/17, Rdnr. 11, juris.

45) VG Hannover, Beschl. v. 25.10.2018 - 4 A 11790/17, Rdnr. 12 , juris; VG Sigmaringen, Beschl. v. 21.8.2018 - 1 K 1825/18, ZUR 2018, 631, 632.

46) VG Hannover, Beschl. v. 25.10.2018 - 4 A 11790/17, Rdnr. 12, juris.

47) OVG Münster, Beschl. v. 6.12.2018 - 8 D 62/18.AK, ZUR 2019, 97, 99 . 
widrigen Regelungslücke analog anzuwenden, weil man ansonsten hinter dem ausdrücklichen Willen des Gesetzgebers zurückbleiben würde. In der von diesem erwähnten BVerwG-Entscheidung seien weder die Zulässigkeit noch Begründetheit des Rechtsbehelfs vom tatsächlichen Bestehen oder der konkreten Möglichkeit einer SUP-Pflicht abhängig gemacht worden. ${ }^{48}$ Nur so werde dem mit der Zuständigkeitsvorschrift des $\$ 7$ Abs. 2 S. 1 UmwRG verfolgten Zweck umfassend Rechnung getragen. Nichts deute darauf hin, dass der Gesetzgeber zwischen SUP-pflichtigen und nicht SUP-pflichtigen Luftreinhalteplänen habe unterscheiden und es dadurch auf das praktisch nicht handhabbare prognostische Kriterium der Rahmensetzung des Luftreinhalteplans in seiner künftigen Gestalt habe ankommen lassen wollen. ${ }^{49}$ Dieser Argumentation hat sich zwischenzeitlich der VGH Mannheim im Ergebnis angeschlossen. ${ }^{50}$ Weil anders als bei der Entscheidung des 7. Senats des BVerwG zum prokuratorischen Klagerecht einer Umweltschutzvereinigung unter Rekurs auf die Gesetzesmaterialien ggf. eine nicht erkannte Gesetzeslücke angenommen werden kann, ist dieses methodische Vorgehen gegenüber der Rechtbehelfsbefugnis nach Maßgabe des $\$ 42$ Abs. 2 HS 2 VwGO, wie sie vom VG Sigmaringen in Erwägung gezogen wird, ${ }^{51}$ vorzuziehen. ${ }^{52} \mathrm{Im}$ Übrigen herrscht Einigkeit, dass auch das Fortschreiben eines Luftreinhalteplans, d.h. dessen begehrte Änderung, die man auch als teilweises Unterlassen für notwendig gehaltener Maßnahmen einordnen könne, rechtsbehelfsfähig ist. ${ }^{53}$

\subsection{Ausnahme: $\int 1$ Abs. 1 S. 1 Nr. 4 HS 2 UmwRG}

Nach $₫ 1$ Abs. 1 S. 1 Nr. 4 HS 2 UmwRG sind von der Rechtsbehelfsfähigkeit solche Pläne und Programme ausgenommen, über deren Annahme durch formelles Gesetz entschieden wird. Bereits aus der Formulierung ,formelles Gesetz" folgt, dass sich diese Ausnahme ausschließlich auf Parlamentsgesetze, nicht jedoch auf Satzungen oder Rechtsverordnungen bezieht. ${ }^{54}$ Bei den Legalplanungen kann es sich sowohl um solche des Bundes- als auch des Landesgesetzgebers handeln. ${ }^{55}$ In den Materialien wurde diese Ausnahme damit begründet, dass in Art. 9 Abs. 3 AK im Unterschied $z u$ dessen Abs. 2 von Handlungen bzw. Unterlassungen von Behörden die Rede ist, nach der Begriffsbestimmung in Art. 2 Nr. 2 S. 2 AK darunter aber keine in gesetzgebender Eigenschaft handelnden Gremien oder Einrichtungen fallen. ${ }^{56}$ Bislang werden so von dem UmwRG SUP-pflichtige Verkehrswegeplanungen auf Bundesebene (Anlage zu $\$ 1$ Abs. 1 S. 2 FStrAbG), SUPpflichtige Bundesbedarfspläne nach $\$ 12$ e EnWG oder die Festlegung von Standortregionen für die über- und untertägige Erkundung für Endlager atomarer Abfälle $(\$ 15$ Abs. 2, $\int 17$ Abs. 2 StandAG) ausgenommen. ${ }^{57}$ Allerdings wird bei Letzteren den Betroffenen vor der Übermittlung des Vorschlags an das Bundesministerium für Umwelt gem. $\int 17$ Abs. 3 StandAG eine auf eine Instanz beschränkte Rechtsschutzmöglichkeit durch Schaffung eines Bescheids durch das Bundesamt für die Sicherheit der nuklearen Entsorgung geschaffen, in welchem dieses feststellt, ob das bisherige Standortauswahlverfahren nach den Regelungen dieses Gesetzes durchgeführt wurde und der Auswahlvorschlag diesem entspricht. ${ }^{58}$

\subsection{Ausnahme: $\int 1$ Abs. 1 S. 3 UmwRG}

Im Zuge der UmwRG-Novelle wurde auch $\$ 1$ Abs. 1 S. 3 UmwRG eingeführt, wonach von diesem Gesetz $\$ 44 \mathrm{a}$ VwGO sowie $₫ 17$ Abs. 3 S. 3-5 und $\ 19$ Abs. 2 S. 5-7 StandAG unberührt bleiben. Entsprechendes gilt gem. $\$ 1$ Abs. 1 S. 1 Nr. 3 UmwRG für $\$ 15$ Abs. 3 S. 2 NABEG ${ }^{59}$, \17a Abs. 5 S. 1 EnWG, $\int 6$ Abs. 9 S. 1 WindSeeG ${ }^{60}, \S 47$ Abs. 4, $\$ 49$ Abs. 3 UVPG und andere entsprechende Vorschriften. Dabei handelt es sich um Vorschriften, nach denen die dort erwähnten Entscheidungen nicht direkt rechtsschutzfähig sind. Nach $₫ 15$ Abs. 3 S. 2 NABEG kann die Bundesfachplanung nur im Rahmen des Rechtsbehelfsverfahrens gegen die Zulassungsentscheidung für die jeweilige Ausbaumaßnahme überprüft werden. ${ }^{61}$ Entsprechendes gilt für den Bundesfachplan Offshore $(\mathbb{S} 17$ Abs. 5 S. 1 EnWG) und für den Flächenentwicklungsplan zur Entwicklung und Förderung der Windenergie auf See (\$6 Abs. 9 S. 1 WindSeeG). Gem. \47 Abs. 4 UVPG kann die Linienbestimmung nach $\$ 16$ Abs. 1 FStrG und die Linienbestimmung nach $\$ 13$ Abs. 1 WaStrG nur im Rahmen des Rechtsbehelfsverfahrens gegen die nachfolgende Zulassungsentscheidung überprüft werden. Gleiches gilt gem. $₫ 49$ Abs. 3 UVPG für das Ergebnis des Raumordnungsverfahrens nach $\$ 15$ ROG. Weiterhin bestimmt $\$ 48$ S. 2 UVPG, dass SUP-pflichtige Raumordnungspläne, die nach Anlage 5 Nr. 1.5 oder Nr. 1.6 Flächen für die Windenergienutzung oder für den Abbau von Rohstoffen aufweisen, nicht nach $\$ 1$ Abs. 1 S. 1 Nr. 4 UmwRG rechtsbehelfsfähig sind. ${ }^{62}$

\subsection{Bewertung}

Durch $\int 1$ Abs. 1 S. 1 Nr. 4 UmwRG wurden die möglichen Streitgegenstände von Umweltrechtsbehelfen deutlich erweitert. Dadurch kann eine Fülle umweltbezogener Pläne einer gerichtlichen Kontrolle zugeführt werden, die - wie ein Blick auf Anlage 5 zum UVPG zeigt - nach ihrem Regelungsgehalt, ihrer Rechtsform, ihrer Konkretisierungstiefe und ihrem Anforderungsprofil durchaus heterogen sind ${ }^{63}$ Fortan ist bei Bebauungsplänen sorgfältig darauf zu achten, ob sie $\$ 1$ Abs. 1 S. 1 Nr. 1 oder Nr. 4 UmwRG unterfallen, denn nur bei Letzteren ist die gerichtliche Überprüfungsbefugnis auf umweltbezogene Vorschriften

48) OVG Münster, Beschl. v. 6.12.2018 - 8 D 62/18.AK, ZUR 2019, 97, 100 .

49) OVG Münster, Beschl. v. 6.12.2018 - 8 D 62/18.AK, ZUR 2019, 97, 100.

50) VGH Mannheim, Urt. v. 18.3.2019 - 10 S 1977/18, NVwZ 2019, 813, 814 .

51) VG Sigmaringen, Beschl. v. 21.8.2018 - 1 K 1825/18, ZUR 2018, 631, 633 .

52) Allgemein eine analoge Rechtsanwendung ablehnend Bunge, in: ders., UmwRG, 2. Aufl. 2019, \$1 Rdnr. 155.

53) VG Hamburg, Beschl. v. 7.8.2018 - 7 K 3876/18 Rdnr. 8 ff., juris; VG Hannover, Beschl. v. 25.10.2018 - 4 A 11790/17, Rdnr. 15, juris; VG Sigmaringen, Beschl. v. 21.8.2018 - $1 \mathrm{~K}$ 1825/18, ZUR 2018, 631, 633; s. auch OVG Münster, Beschl. v. 6.12.2018-8 D 62/18.AK, ZUR 2019, 97, 98.

54) BT-Drs. 18/9526, S. 35.

55) Fellenberg/Schiller, in: Landmann/Rohmer, Umweltrecht, 90. EL 6/2019, UmwRG \$ 1 Rdnr. 97.

56) BT-Drs. 18/9526, S. 35; zur Völkerrechtskonformität auch Schlacke, in: Gärditz, VwGO, 2. Aufl. 2018, \1 Rdnr. 46.

57) Fellenberg/Schiller, in: Landmann/Rohmer, Umweltrecht, 90. EL 6/2019, UmwRG \1 Rdnr. 97; Schlacke, in: Gärditz, VwGO, 2. Aufl. 2018, \$1 UmwRG Rdnr. 46.

58) Dazu Wollenteit, NuR 2018, 818, $820 \mathrm{f}$.

59) Netzausbaubeschleunigungsgesetz Übertragungsnetz (NABEG) v. 28. Juli 2011, BGB1. I S. 1690; zuletzt geändert durch Artikel 2 des Gesetzes v. 13.5.2019, BGBl. I S. 706.

60) Gesetz zur Entwicklung und Förderung der Windenergie auf See (WindSeeG) v. 13. 10.2016, BGB1. I S. 2258, 2310; zuletzt geändert durch Artikel 21 des Gesetzes v. 13.5.2019, BGBl. I S. 706.

61) Kritisch zu dieser Entscheidung des Gesetzgebers, weil die Bundesfachplanung gegenüber der Planfeststellungsbehörde wie ein verbindliches Raumordnungsziel wirkt, Durner, EurUP 2018 , 142, 152f. Zur Völker- und Unionsrechtskonformität Recht, Rechtsschutz im Rahmen des beschleunigten Stromnetzausbaus, 2019, S. $236 \mathrm{ff}$.

62) Zur Interpretation dieser Regelung Bunge, in: ders., UmwRG, 2. Aufl. 2019, \&1 Rdnr. $117 \mathrm{ff}$.

63) Durner, EurUP 2018, 142 f. 
beschränkt. ${ }^{64}$ Die langen Ausführungen der Gerichte zur Rechtsbehelfsfähigkeit von Luftreinhalteplänen sind weniger Folge schlechter Gesetzgebung, sondern basieren auf der vom BVerw $G$ zwischenzeitlich vorgenommenen engen Auslegung des $₫ 1$ Abs. 1 S. 1 Nr. 1 UmwRG. Zunehmend gelangt man zu der Erkenntnis, dass sich die Beschränkung des $\$ 1$ Abs. 1 S. 1 Nr. 4 UmwRG auf SUP-pflichtige Pläne und Programme aus völker- und unionsrechtlicher Sicht als zu kurz gegriffen erweist. So müssen unter Zugrundelegung der Protect-Entscheidung des EuGH z.B. auch Landschaftsschutzgebiete, die einen Bezug zum unionalen Umweltrecht, etwa der Wasserrahmenrichtlinie aufweisen, im Hinblick auf Art. 9 Abs. 3 AK umweltrechtsbehelfsfähig sein. ${ }^{65}$ Ähnliches gilt für den mangels Erwähnung in der Anlage 5 zum UVPG nicht aufgeführten wasserrechtlichen Bewirtschaftungsplan. ${ }^{66}$ Außerdem folgt aus der EuGH-Rechtsprechung zum slowakischen Braunbären II, dass Pläne, auf die gem. Art. 6 Abs. 1 lit. b) AK das Verfahren nach Art. 6 Abs. 3 FFH-Richtlinie ${ }^{67}$ Anwendung findet, nicht Art. 9 Abs. 3 AK, sondern Art. 9 Abs. 2 AK zuzuordnen sind mit der Folge, ${ }^{68}$ dass bei ihnen auch die Einhaltung anderer als nur umweltbezogener Vorschriften zu prüfen ist. ${ }^{69}$

Auch wird sich erst zeigen müssen, ob die Herausnahme bestimmter SUP-pflichtiger Pläne und Programme durch $\$ 1$ Abs. 1 S. 1 Nr. 4 UmwRG und der Verweis auf inzidente Rechtsschutzmöglichkeiten auf einer späteren Stufe in ihrer Gesamtheit als unions- und völkerrechtskonform angesehen werden kann. ${ }^{70}$ Das ACC meinte hierzu in seinem Bericht vom 31.7.2017, dass der Anwendungsbereich von ,umweltbezogenen“ Plänen und Programmen weiter als der Anwendungsbereich der SUP sein könne. ${ }^{71}$ Lediglich in Bezug auf Pläne, die keiner Pflicht zur Vorbereitung einer SUP unterliegen, merkte es an, dass Art. 9 Abs. 3 AK keine spezifischen Anforderungen zum Zeitpunkt ihrer Angreifbarkeit mache. Deshalb akzeptierte es, dass bei derartigen gegen umweltbezogene Vorschriften verstoßenden Plänen und Programmen die Möglichkeit der inzidenten Überprüfung im Rahmen des gerichtlichen Rechtsschutzes einer darauf basierenden Entscheidung ,ausreichen kann, aber nicht muss - zum Beispiel kann es Pläne oder Programme geben, zu denen es keine nachgelagerten Entscheidungen gibt, die überprüft werden könnten" oder könnten ,die Elemente des Plans oder Programms, die gegen nationale Umweltgesetze verstoßen, außerhalb des Anwendungsbereichs der Prüfung einer darauf basierenden Entscheidung liegen". ${ }^{72}$ Außerdem akzeptierte das ACC hinsichtlich $\$ 1$ Abs. 1 S. 3 Nr. 3 UmwRG, dass es bestimmte Pläne und Programme geben kann, bei denen der spätere inzidente Rechtsschutz ausreichend ist. Weil keine spezifischen Informationen in Bezug auf die Unzulänglichkeiten dieses Rechtsschutzes vorgelegt wurden, sah sich das ACC zur Annahme eines Verstoßes gegen die Entscheidung V/9h außerstande, wies aber auf verbleibende Unsicherheiten hin. ${ }^{73}$

\section{4. $\$ 1$ Abs. 1 S. 1 Nr. 5 UmwRG}

Darüber hinaus sind nunmehr gem. $₫ 1$ Abs. 1 S. 1 Nr. 5 UmwRG Verwaltungsakte oder öffentlich-rechtliche Verträge umweltrechtsbehelfsfähig, durch welche andere als den Nrn. 1-2b unterfallende Industrieanlagen oder Infrastrukturmaßnahmen unter Anwendung umweltbezogener Rechtsvorschriften des Bundesrechts, des Landesrechts oder unmittelbar geltender Rechtsakte der Europäischen Union zugelassen werden. In den Gesetzesmaterialien hierzu wird darauf verwiesen, dass der Begriff des Vorhabens in Anlehnung an $\$ 2$ Abs. 2 UVPG (= heute $\$ 2$ Abs. 4 UVPG), jedoch ohne Bezugnahme auf die Anlage 1 des UVPG, $\mathrm{zu}$ verstehen sei. ${ }^{74} \mathrm{Zu}$ denken sei etwa an die Errichtung und den Betrieb einer technischen oder sonstigen Anlage, den Bau einer anderen Anlage oder die Durchführung ei- ner sonstigen, mit einem Eingriff in Natur und Landschaft verbundenen Maßnahme einschließlich jeweils deren Änderung oder Erweiterung. ${ }^{75}$ Erfasst würden auch besondere Ausgestaltungen der fachrechtlichen Zulassungsentscheidungen, etwa Vorbescheide und Teilgenehmigungen, aufgrund ihres VA-Charakters. ${ }^{76}$ Die Umweltrechtsbehelfsfähigkeit auch eines Vorbescheids trägt dem Umstand Rechnung, dass dieser einen Teil der späteren Genehmigungsentscheidung vorwegnimmt und die Behörde grundsätzlich bindet. ${ }^{77}$

Nunmehr findet das UmwRG aufimmissionsschutzrechtliche (Änderungs-)Genehmigungen, etwa für eine einzelne Windenergieanlage, die nicht nach Nr. 1.6 der Anlage zum UVPG UVP-pflichtig und auch nicht in der Spalte $c$ des Anhangs 1 der 4. BImSchV mit dem Buchstaben G gekennzeichnet ist, ${ }^{78}$ oder auf wasserrechtliche Erlaubnisse, die nicht mit einem Vorhaben i. S.d. IED-Richtlinie verbunden sind (s. Nr. 2), Anwendung. Auf Baugenehmigungen und Befreiungen nach $₫ 31$ Abs. 2 BauGB wird sie angewandt, soweit das jeweilige Vorhaben unter Anwendung umweltbezogener Vorschriften zugelassen wird. ${ }^{79}$ Auch die Genehmigung der Umwandlung von Waldbewirtschaftung in eine andere Nutzungsart stellt als eine sonstige in Natur und Landschaft eingreifende Maßnahme ebenfalls eine rechtsbehelfsfähige Entscheidung dar. ${ }^{80}$ Da sich $\$ 1$ Abs. 1 S. 1 Nr. 5 UmwRG nur auf Verwaltungsakte oder öffentlich-rechtliche Verträge bezieht, lassen sich darunter

64) Dazu, dass den Verbänden eine Differenzierung zwischen UVPund SUP-pflichtigen Bebauungsplänen nicht erspart wird, weil sie bei nicht UVP-pflichtigen Bebauungsplänen die Anforderungen des $\$ 2$ Abs. 1 S. 1 Nr. 3b, S. 2 UmwRG erfüllen müssen, $M a-$ ger, EurUP 2018, 50, 55.

65) Schlacke, NVwZ 2019, 1392, 1398; dazu, dass die Vorstellung einer ausreichenden Umsetzung des Art. 9 Abs. 3 AK durch die Protect-Entscheidung hinfällig geworden sein dürfte, Durner, EurUP 2018, 142, 143; s. auch Teßmer, in: FS für Baumann, 2019, S. $349,367 \mathrm{ff}$.

66) S. dazu auch Durner, EurUP 2018, 142, 150.

67) Richtlinie 92/43/EWG des Rates vom 21.5.1992 zur Erhaltung der natürlichen Lebensräume sowie der wildlebenden Tiere und Pflanzen, ABl. 1992 L 206, S. 7.

68) Bunge, in: ders., UmwRG, 2. Aufl. 2019, $₫ 1$ Rdnr. 157; Franzius, NVwZ 2018, 219, 220.

69) Fischer-Hüftle, NuR 2018, 735, 740.

70) Ohne Bedenken gegenüber einer Konzentration des Rechtsschutzes Schieferdecker, in: Hoppe/Beckmann/Kment, UVPG/ UmwRG, 5. Aufl. 2018, \$1 UmwRG Rdnr. 95; für eine Unanwendbarkeit des \48 S. 2 ROG aufgrund der Protect-Rechtsprechung Erbguth, DVBl. 2018, 897, 905.

71) ACC, ZUR 2018, 23, 26

72) ACC, ZUR 2018, 23, 26; daneben stellt sich die Frage, ob nicht aus der EuGH-Rechtsprechung zu Art. 9 Abs. 3 AK strengere Maßstäbe folgen können; hierzu näher bezogen auf $\$ 15$ Abs. 3 S. 2 NABEG, Recht, Rechtsschutz im Rahmen des beschleunigten Stromnetzausbaus, 2019, S. $236 \mathrm{ff}$

73) ACC, ZUR 2018, 23, 26

74) BT-Drs. 18/9526, S. 36.

75) BT-Drs. 18/9526, S. 36.

76) BT-Drs. 18/9526, S. 36; s. dazu auch Schieferdecker, in: Hoppe/ Beckmann/Kment, UVPG/UmwRG, 5. Aufl. 2018, \$1 UmwRG Rdnr. 33.

77) OVG Lüneburg, Urt. v. 25. 10.2018 - 12 LB 118/16, Rdnr. 148, juris.

78) OVG Greifswald, Beschl. v. 8.5.2018 - 3 M 22/16, Rdnr. 58, juris; s. auch OVG Berlin-Brandenburg, Beschl. v. 22.8.2018 - OVG 11 S 10.18, Rdnr. 4, juris; VGH München, Beschl. v. 5. 4.2019 - 22 CS 18.2572, 22 CS 19.23, Rdnr. 41, juris.

79) VG Neustadt a.d.W., Beschl. v. 26.9.2019 - 5 L 963/19.NW, Rdnr. 16 ff., juris; dazu, dass bei Baugenehmigungen genau darauf zu achten ist, ob umweltbezogene oder andere Vorschriften in Rede stehen, VGH München, Beschl. v. 11.4.2018 - 2 CS 18.198, NuR 2019, 483.

80) OVG Lüneburg, Urt. v. 21.8.2018 - 10 LB 34/18, ZUR 2019, 42. 
Realakte ohne eine vorherige Zulassungsentscheidung, wie etwa das Fällen einer Platane oder die Beseitigung eines Teils einer Hecke, nicht subsumieren. ${ }^{81}$ Ebenso wenig sind untergesetzliche Rechtsvorschriften, etwa die Festlegung von Flugrouten oder Naturschutzgebieten durch Rechtverordnung, nach $₫ 1$ Abs. 1 S. 1 Nr. 5 UmwRG umweltrechtsbehelfsfähig. ${ }^{82}$ Zwischenzeitlich erging eine ganze Reihe von Gerichtsentscheidungen zu dieser Neuregelung. In deren Mittelpunkt stehen vor allem Fragen, die sich aus der Fokussierung dieser Regelung auf die Zulassung von Vorhaben ergeben sowie das Verhältnis von $\$ 1$ Abs. 1 S. 1 Nr. 1 und Nr. 5 UmwRG betreffen.

\subsection{Rechtsprechung mit Bezügen zum Vorhabenbegriff}

Schon früh entschieden das VG Düsseldorf und das VG Schleswig, dass die straßenverkehrsrechtliche Zulassung eines Fahrzeugs keinen Verwaltungsakt in Bezug auf ein Vorhaben i.S.d. $\int 1$ Abs. 1 S. 1 Nr. 5 UmwRG enthält. Schon nach dem allgemeinen Sprachgebrauch verstehe man unter einem „Vorhaben“ eine Absicht, ein Projekt, ein Unterfangen oder Unternehmen, mithin ein planerisches Handeln. ${ }^{83}$ Im juristischen Sprachgebrauch werde der Vorhabenbegriff regelmäßig im Planungsrecht hinsichtlich der Umsetzung planerischer Maßnahmen verwendet, die sich auf Anlagen beziehen. ${ }^{84}$ Bestätigt werde dies durch einen Blick auf die Erwähnung, ,andere[r] als in den Nummern 1 bis $2 b$ genannte[n] Vorhaben" in der Nr. 5, die sich allesamt auf planerische Maßnahmen in Bezug auf ortsfeste Anlagen oder auf solche mit ortsspezifischen Eingriffen in Natur und Landschaft beziehen. ${ }^{85}$ Angesichts der erneuten Verwendung des Begriffs in Nr. 5 seien darunter nur solche Vorhaben zu fassen, die denjenigen der Nrn. 1-2b vergleichbar sind ${ }^{86}$ Ein Blick auf die Materialien ergebe, dass der Vorhabenbegriff wie in $\$ 2$ Abs. 4 UVPG ohne die Bezugnahme auf die Anlage $1 \mathrm{zu}$ verstehen ist. ${ }^{87}$ Aufgrund der Abweichung der Umweltrechtsbehelfe vom Individualrechtsschutz sowie der listenmäßigen Aufzählung könne gerade nicht davon ausgegangen werden, dass nunmehr jede umweltbezogene Behördenentscheidung umweltrechtsbehelfsfähig sein solle. ${ }^{88}$ Gegenüber den anderen in $\$ 1$ Abs. 1 S. 1 Nrn. $1-2 b$ UmwRG genannten Vorhaben wiesen produkt- und straBenverkehrsrechtliche Einzelzulassungen von Kfz nur geringfügige Auswirkungen auf die Umwelt auf und sei das Interesse der Allgemeinheit am Umweltschutz daher als gering einzustufen. ${ }^{89}$ Derartige Behördenentscheidungen enthalten keine planerischen Maßnahmen, die physisch und in Bezug auf einen spezifischen Ort oder ein Gebiet unmittelbar in Natur und Landschaft eingreifen. ${ }^{90}$ Aus Umweltperspektive bestehe vor allem bei Typengenehmigungen für eine unbestimmte Vielzahl von Fahrzeugen ein Allgemeininteresse an der Einhaltung des Umweltschutzes. ${ }^{91}$ Unter Hinweis auf die Gesetzgebungsgeschichte, in der diese Thematik sehr wohl zur Sprache gebracht, aber nicht vom Gesetzgeber aufgegriffen wurde, bleibe allerdings nichts anderes übrig, als die Umweltrechtsbehelfsfähigkeit dieser Entscheidungen zu verneinen. ${ }^{92}$

Zwar ergeht eine raumordnungsrechtliche Zielabweichung in Gestalt eines Verwaltungsakts. Trotzdem verneinte der VGH Kassel aber wiederum die Einschlägigkeit des $₫ 1$ Abs. 1 S. 1 Nr. 5 UmwRG mangels Zulassung eines Vorhabens mit folgender Begründung: Unter die sonstigen Anlagen nach $₫ 2$ Abs. 4 lit. b) UVPG falle zwar der Bau nichttechnischer Anlagen; die sonstigen in Natur und Landschaft eingreifenden Maßnahmen seien an den Eingriffsbegriff in $\int 14$ BNatSchG angelehnt. ${ }^{93}$ Die Zielabweichung lasse jedoch weder den Bau einer Anlage noch direkte Eingriffe oder Arbeiten im Raum zu, sondern beschränke sich auf die teilweise Suspendierung von Zielen des Raumordnungsplans. ${ }^{94}$

Das OVG Berlin-Brandenburg stellte sich hinsichtlich einer pflanzenschutzrechtlichen Genehmigung nach $\$ 18$
Abs. 2 PflSchG zwar auf den Standpunkt, dass diese weder den Bau einer sonstigen Anlage betreffe noch zu einer Veränderung der Gestalt oder Nutzung von Grundflächen oder Veränderungen des mit der belebten Bodenschicht in Verbindung stehenden Grundwasserspiegels führe. Im Unterschied zu den vorherigen Gerichtsentscheidungen sah es sich aber nicht an einer erweiternden Auslegung des $₫ 1$ Abs. 1 S. 1 Nr. 5 UmwRG gehindert. Angesichts des Willens des Gesetzgebers zur vollständigen Umsetzung von Art. 9 Abs. 3 AK, dessen Anwendungsbereich sich allgemein auf gegen umweltbezogene Bestimmungen verstoßende behördliche Handlungen oder Unterlassungen bezieht, der zu einer weiten Auslegung neigenden Spruchpraxis des ACC, der Konzeption des $\$ 1$ Abs. 1 S. 1 Nr. 5 UmwRG als Auffangtatbestand sowie der EuGH-Rechtsprechung zu Art. 9 Abs. 3 AK sei auch eine solche Entscheidung als umweltrechtsbehelfsfähig zu qualifizieren. ${ }^{95}$

Ferner mussten die Verwaltungsgerichte prüfen, inwieweit $\$ 1$ Abs. 1 S. 1 Nr. 5 UmwRG bei $\$ 15$ Abs. 2 S. 2 BImSchG Relevanz erlangt. Danach hat die Behörde unverzüglich nach Anzeige einer Änderung zu prüfen, ob diese

81) OVG Münster, Beschl. v. 8.3.2019 - 8 B 1651/18, NuR 2019, 494; zu Realakten insgesamt Fellenberg/Schiller, in: Landmann/ Rohmer, Umweltrecht, 90. EL 6/2019, UmwRG \$ 1 Rdnr. 112; Schlacke, in: Gärditz, VwGO, 2. Aufl. 2018, \1 UmwRG Rdnr. 53.

82) Dikaios, Überindividueller Umweltrechtsschutz am Beispiel der altruistischen Verbandsklage in der deutschen, griechischen und europäischen Rechtsordnung, 2018, S. 522; Schlacke, in: Gärditz, VwGO, 2. Aufl. 2018, \$1 UmwRG Rdnr. 53.

83) VG Düsseldorf, Urt. v. 24.1.2018 - 6 K 12341/17, Rdnr. 91 ff., juris.

84) VG Düsseldorf, Urt. v. 24.1.2018 - 6 K 12341/17, Rdnr. $96 \mathrm{ff}$, juris; ähnlich VG Schleswig, Urt. v. 13.12.2017 - 3 A 38/17, ZUR 2018, 239 ff.; s. auch EuGH, Urt. v. 29.7.2019 - C-411/17, ECLI:EU:C:2019:622, Rdnr. 62 - Inter-Environnement Wallonie und Bond Beter Leefmilieu Vlaanderen, wonach unter dem Begriff, „Projekt“ Arbeiten oder Eingriffe zu verstehen sind, welche den materiellen Zustand eines Platzes verändern.

85) VG Düsseldorf, Urt. v. 24.1.2018 - 6 K 12341/17, Rdnr. 91, juris.

86) VG Düsseldorf, Urt. v. 24.1.2018 - 6 K 12341/17, Rdnr. 96, juris; s. auch VG Schleswig, Urt. v. 13.12.2017 - 3 A 38/17, ZUR 2018, 239, 240 .

87) VG Düsseldorf, Urt. v. 24.1.2018 - 6 K 12341/17, Rdnr. 140, juris; s. auch VG Schleswig, Urt. v. 13.12.2017 - 3 A 38/17, ZUR 2018, 239, 240

88) VG Düsseldorf, Urt. v. 24.1.2018 - 6 K 12341/17, Rdnr. 110, juris.

89) VG Düsseldorf, Urt. v. 24.1.2018 - 6 K 12341/17, Rdnr. 116, juris.

90) VG Düsseldorf, Urt. v. 24.1.2018 - 6 K 12341/17, Rdnr. 89, juris; s. auch VG Schleswig, Urt. v. 13.12.2017 - 3 A 38/17, ZUR 2018, 239, 240. Bunge, in: ders., UmwRG, 2. Aufl. 2019 $\$ 1$ Rdnr. 139 f., hält es für möglich, bestimmte mobile Aktivitäten unter den Vorhabenbegriff zu fassen, nicht jedoch Entscheidungen in Bezug auf Produkte.

91) VG Düsseldorf, Urt. v. 24.1.2018 - 6 K 12341/17, Rdnr. 116 ff., juris, die jedoch nicht streitgegenständlich waren; allgemein zu Neuerungen im Typengenehmigungsrecht Schmidt-Kötters/Geber, NVwZ 2019, $1809 \mathrm{ff}$.

92) VG Düsseldorf, Urt. v. 24.1.2018 - 6 K 12341/17, Rdnr. 119 ff., juris; dazu, dass andernfalls eine unzulässige richterliche Rechtsfortbildung vorgenommen würde, VG Schleswig, Urt. v. 13.12.2017 - 3 A 38/17, ZUR 2018, 239, 241; ebenso Fellenberg/ Schiller, in: Landmann/Rohmer, Umweltrecht, 90. EL Juni 2019, UmwRG $\$ 1$ Rdnr. 102 zur Frage der völkerrechtskonformen Auslegung von Betriebszulassungen.

93) VGH Kassel, Beschl. v. 15.8.2019 - 4 B 1303/19, Rdnr. 46 f., juris.

94) VGH Kassel, Beschl. v. 15.8.2019 - 4 B 1303/19, Rdnr. 47 f., juris; ebenso Fellenberg/Schiller, in: Landmann/Rohmer, Umweltrecht, 90. EL Juni 2019, UmwRG \$1 Rdnr. 111.

95) OVG Berlin-Brandenburg, Beschl. v. 17.5.2019 - OVG $11 \mathrm{~S}$ 40.19, Rdnr. 7, juris. 
einer Genehmigung bedarf. Sobald die Behörde mitteilt, dass eine solche nicht notwendig ist oder sich nicht fristgemäß äußert, darf der Vorhabenträger mit der Änderung beginnen. Zutreffend stellte der VGH München zunächst heraus, dass die Freistellungsbescheinigung keine Zulassungsentscheidung i.S.d. $\int 1$ Abs. 1 S. 1 Nr. 5 UmwRG enthält, zumal Anzeigeverfahren von der Legaldefinition der Zulassungsentscheidung in $₫ 2$ Abs. 6 Nr. 1 a. E. UVPG ausgeklammert sind. ${ }^{96}$ Inhaltlich wird durch diese zwar verbindlich über die fehlende Genehmigungsbedürftigkeit, nicht aber die Zulassung des Vorhabens entschieden. ${ }^{97}$ In der Freistellungsentscheidung liegt jedoch ein behördlicher Verzicht auf eine immissionsschutzrechtliche Genehmigung, so dass das Unterlassen einer $\$ 16 \mathrm{BImSchG-Ge-}$ nehmigung gem. \$1 Abs. 1 S. 2 UmwRG geltend gemacht werden kann. Selbst wenn nach dem Wortlaut dieser Regelung unklar ist, ob mangels Genehmigungsantrags in der Erteilung einer Freistellungserklärung ein behördliches Unterlassen der Genehmigungserteilung liege, komme diese Regelung nach ihrem Sinn und Zweck auch dann zum Tragen, ${ }^{98}$ denn ausweislich der Gesetzesmaterialien soll diese Regelung greifen, wenn ein Zulassungsverfahren nicht durchgeführt wurde, obwohl es hätte durchgeführt werden müssen. ${ }^{99}$ Ferner sei eine solche Auslegung im Hinblick auf Art. 9 Abs. 3 AK geboten. ${ }^{100}$ Diese Rechtsprechung lässt sich auf Freistellungen nach $\ 43 \mathrm{f}$ EnWG oder \25 NABEG übertragen. ${ }^{101}$

\subsection{Verhältnis zwischen $\ 1$ Abs. 1 S. $1 \mathrm{Nr} .1$ und Nr. 5 UmwRG}

Probleme bereitet schließlich das Verhältnis zwischen $\int 1$ Abs. 5 S. 1 Nr. 1 und Nr. 5 UmwRG. Im Ausgangspunkt wird angenommen, dass aufgrund der Formulierung ,durch die andere Vorhaben als in den Nrn. 1-2b zugelassen werden" beide Vorschriften zueinander in einem Exklusivitätsverhältnis stehen. ${ }^{102} \mathrm{Da}$ an die Differenzierung zwischen den einzelnen Entscheidungen unterschiedliche Rechtsfolgen (z.B. in $\$ 2$ Abs. 1 UmwRG) anknüpfen, müssten sich die verschiedenen Entscheidungstypen untereinander ausschließen. ${ }^{103}$ Zutreffend wird jedoch davon ausgegangen, dass zur Vermeidung von Rechtsschutzlücken in Konstellationen, in denen ein Vorhaben einer UVP-Vorprüfung unterliegt und die UVP-Pflicht von der zuständigen Behörde rechtmäßig verneint wurde, auffangweise dennoch auf $\$ 1$ Abs. 1 S. 1 Nr. 5 UmwRG zu rekurrieren ist. ${ }^{104}$ Trotz des Wortlauts sei es nicht ausgeschlossen, bei einer immissionsschutzrechtlichen Genehmigung nach $\$ 19$ BImSchG auf $\$ 1$ Abs. 1 S. 1 Nr. 5 UmwRG zurückzugreifen. ${ }^{105}$ Mit den Worten des VGH Mannheim wäre es sinnwidrig, wenn bei Kleinvorhaben, die schon aufgrund ihrer Art, geringen Größe oder geringen Leistung keiner Pflicht zur UVP-Vorprüfung unterliegen, ,eine vollständige gerichtliche Kontrolle zumindest der Einhaltung umweltbezogener Rechtsvorschriften verlangt werden könnte, [...] demgegenüber [...] anerkannte Umweltvereinigungen bei einem vorprüfungspflichtigen und damit im Ansatz mit größeren Risiken für die Umweltbelange verbundenen Vorhaben nur eine eingeschränkte Kontrolle im Hinblick auf die insoweit relevanten erheblichen nachteiligen Umweltauswirkungen verlangen“" könnten. ${ }^{106}$

\subsection{Bewertung}

Alles in allem zeigt sich, dass die Rechtsprechung zum Verhältnis zwischen $\$ 1$ Abs. 1 S. 1 Nr. 1 und Nr. 5 UmwRG eine Folge der Rechtsprechung des BVerwG zur Nr. 1 ist, wonach bereits der Tatbestand der UVP-Pflichtigkeit umfassend im Rahmen der Zulässigkeit zu prüfen ist. Angesichts des engen Vorhabenbegriffs sind auf Seiten der Gerichte Unsicherheiten $\mathrm{zu}$ verzeichnen, ob sie trotz der Gesetzgebungsgeschichte eine erweiternde Auslegung die- ses Tatbestandsmerkmals vornehmen dürfen. Während wenige Stimmen im Schrifttum von der Unions- und Völkerrechtskonformität der engen Fassung des $\ 1$ Abs. 1 S. 1 Nr. 5 UmwRG aufgrund des Ausgestaltungsspielraums der Vertragsparteien überzeugt sind, ${ }^{107}$ äußert der überwiegende Teil Zweifel wegen der generalklauselartigen Fassung des Art. 9 Abs. 3 AK und des mit diesem Abkommen intendierten möglichst weiten Gerichtszugangs. ${ }^{108}$ Da die AK lediglich für das Handeln des Parlaments in gesetzgebender Eigenschaft nicht gilt, werden Bedenken an der Ausklammerung untergesetzlicher Rechtsvorschriften von den Umweltrechtsbehelfen geäußert. ${ }^{109}$ Auch ist noch nicht abschlieBend geklärt, ob eine solche Zulassung in der Verlängerung einer immissionsschutzrechtlichen Genehmigung nach $₫ 18$ Abs. 3 BImSchG gesehen werden kann. Dagegen wird vorgebracht, dass es hier im Kern an einer neuen Zulassung fehlt. ${ }^{110}$ Kürzlich stellte sich jedoch der EuGH auf den Standpunkt, dass eine Verlängerung im Kontext von Modernisierungsmaßnahmen, die damit untrennbar verbunden sind, als Genehmigung eines Projekts eingestuft werden könne. ${ }^{111}$ Auch bejahte das OVG Berlin-Brandenburg plausibel, dass jedenfalls bei einer rechtsfehlerhaft gewährten Fristverlängerung die Umweltrechtsbehelfsfähigkeit wegen Unterlassens der Behörde anzunehmen ist, wenn bei korrekter Verfahrensweise, also einer Ablehnung der Fristverlängerung, ein Antrag auf Durchführung eines neuen Genehmigungsverfahrens hätte gestellt werden müssen. ${ }^{12}$

\section{5. $\$ 1$ Abs. 1 S. 1 Nr. 6 UmwRG}

Schließlich sind Verwaltungsakte über Überwachungsoder Aufsichtsmaßnahmen zur Umsetzung oder Durch-

96) VGH München, Beschl. v. 5.4.2019 - 22 CS 18.2572, 22 CS 19.23, Rdnr. 61, juris.

97) VG Regensburg, Beschl. v. 21.11.2018 - RN 7 S 18.1756 , Rdnr. 55, juris.

98) VGH München, Beschl. v. 5.4.2019 - 22 CS 18.2572, 22 CS 19.23, Rdnr. 61, juris.

99) VGH München, Beschl. v. 5.4.2019 - 22 CS 18.2572, 22 CS 19.23, Rdnr. 61, juris unter Verweis auf BT-Drs. 16/2495, S. 10

100) VGH München, Beschl. v. 5.4.2019 - 22 CS 18.2572, 22 CS 19.23, Rdnr. 61, juris.

101) Schlacke, NVwZ 2019, 1392, 1399; dies./Römling, DVBl. 2019 , $1429,1435$.

102) VG Hannover, Beschl. v. 28.2.2019 - 12 B 6923/18, Rdnr. 69, juris; VG Sigmaringen, Urt. v. 14.11.2018 - 10 K 118/17, Rdnr. 77, juris.

103) VG Sigmaringen, Urt. v. 14.11.2018 - 10 K 118/17, Rdnr. 77, juris.

104) VG Hannover, Beschl. v. 28.2.2019 - 12 B 6923/18, Rdnr. 69 juris; s. auch VGH Mannheim, Urt. v. 20.11.2018 - 5 S 2138/16, Rdnr. 165, juris; a.A. Schieferdecker, in: Hoppe/Beckmann/ Kment, UVPG/UmwRG, 5. Aufl. 2018, \$1 UmwRG Rdnr. 66.

105) VG Hannover, Beschl. v. 28.3.2019 - 4 B 5526/18, Rdnr. 65, juris.

106) VGH Mannheim, Urt. v. 20.11.2018 - Az. 5 S 2138/16, Rdnr. 163, juris.

107) Ruffert, DVB1. 2019, 1033, 1039.

108) Brigola/Heß, NuR 2017, 729, 730; Schlacke, in: Gärditz, VwGO, 2. Aufl. 2018, \$1 UmwRG Rdnr. 55.

109) Franzius, in: Schink/Reidt/Mitschang, UVPG/UmwRG, 2018, \1 UmwRG Rdnr. 26; Pernice-Warnke, DÖV 2017, 846, 853; Schlacke, in: Gärditz, VwGO, 2. Aufl. 2018, \1 UmwRG Rdnr. 55.

110) Schieferdecker, in: Hoppe/Beckmann/Kment, UVPG/UmwRG, 5. Aufl. 2018, \$1 UmwRG Rdnr. 36; Schlacke, NVwZ 2019, 1392, 1399; a.A. OVG Berlin-Brandenburg, Beschl. v. 5.9.2014 - OVG $11 \mathrm{~S}$ 44.14, ZUR 2015, 45, 46f., allerdings zum UmwRG vor seiner Novellierung.

111) EuGH, Urt. v. 29.7.2019 - C-411/17, ECLI:EU:C:2019:622, Rdnr. $71 \mathrm{ff} ., 77 \mathrm{ff}$. - Inter-Environnement Wallonie und Bond Beter Leefmilieu Vlaanderen.

112) OVG Berlin-Brandenburg, Urt. v. 4.9.2019 - OVG 11 B 24.16, Rdnr. 30, juris. 
führung von Entscheidungen nach den Nrn. 1-5, die der Einhaltung umweltbezogener Rechtsvorschriften des Bundesrechts, des Landesrechts oder unmittelbar geltender Rechtsakte der Europäischen Union dienen, aufgrund von Umweltrechtsbehelfen überprüfbar. Da nach Art. 9 Abs. 3 AK Rechtsschutz in Bezug auf Handlungen oder Unterlassungen von Privatpersonen bei einem Verstoß gegen umweltbezogene Rechtsvorschriften möglich sein muss, dagegen aber keine direkten zivilrechtlichen Rechtsbehelfe eröffnet sind, verständigte man sich auf eine Rechtsbehelfsmöglichkeit gegen Behörden, die im Zuge der Überwachung oder sonstiger aufsichtlicher Maßnahmen auf umweltrechtskonforme Zustände bei den Privaten hinwirken können. ${ }^{113}$ Auch nach Meinung des ACC verlangt Art. 9 Abs. 3 AK keinen direkten Rechtsschutz der Mitglieder der Öffentlichkeit in Bezug auf privatwirtschaftliche Akteure, sofern der Rückgriff auf behördliche Verfahren eine wirksame Durchsetzung des Umweltrechts gewährleistet und den Anforderungen aus Art. 9 Abs. 4 AK entspricht. ${ }^{114}$ Wie bereits im vorherigen Heft aufgezeigt, werden künftig aufgrund der europäischen Sammelklage in bestimmten Konstellationen direkte Rechtsschutzmöglichkeiten von Umweltorganisationen gegen Private eröffnet sein. ${ }^{115}$ Indem die Aufsichts- oder Überwachungsmaßnahmen des $\int 1$ Abs. 1 S. 1 Nr. 5 UmwRG den Charakter von Verwaltungsakten i.S.d. $\$ 35 \mathrm{VwVfG}$ aufweisen, da sie insbesondere auf unmittelbare Außenwirkung gerichtet sind, werden von dieser Neuregelung behördeninterne Maßnahmen, etwa Weisungen im Rahmen der Bundesauftragsverwaltung, nicht erfasst. ${ }^{116}$

Da sich der Gesetzeswortlaut auf Aufsichts- oder Überwachungsmaßnahmen bezieht, bedarf es keiner trennscharfen Abgrenzung zwischen diesen. ${ }^{117}$ Der weit auszulegende Begriff der Überwachungsmaßnahme beschränkt sich nicht auf die behördliche Eigenüberwachung (z.B. gem. \$28 UVPG, J\$100f. WHG), sondern erfasst auch behördliche Anordnungen gegenüber dem Vorhabenträger oder Dritten. ${ }^{118}$ Unter Überwachungsmaßnahmen lassen sich in einem weiten Sinne Maßnahmen des Gesetzesvollzugs verstehen, wie z.B. das Einschreiten nach $\$ 16$ Abs. 3 S. 1 SeeAnlV. ${ }^{119}$ Unter Verweis auf die Umsetzungsnotwendigkeiten des Art. 9 Abs. 3 AK sowie die Materialien sprach sich das OVG Hamburg zutreffend gegen eine $z u$ enge Auslegung dieser Regelung aus, wonach damit nur Maßnahmen zur Durchsetzung der aus der Genehmigung selbst hervorgehenden Vorgaben gemeint seien. ${ }^{120}$ Als weitere Beispiele für derartige aufsichtsrechtliche Maßnahmen seien Beseitigungsanordnungen oder Nutzungsuntersagungen, aber auch nachträgliche Anordnungen genannt. ${ }^{121}$

\section{Zwischenfazit zum Listenprinzip}

Trotz der Vergrößerung des Anwendungsbereichs des UmwRG in $\$ 1$ Abs. 1 UmwRG steht die Klärung der Frage, ob durch die enumerative Aufzählung der umweltrechtsbehelfsfähigen Gegenstände Art. 9 Abs. 3 AKK hinreichend umgesetzt wurde, noch aus. Das ACC hob in seinem Bericht vom 31.7.2017 die deutliche Erweiterung der Rechtsschutzmöglichkeiten für Umweltorganisationen aufgrund der UmwRG-Novelle 2017 hervor, betonte aber zugleich an einigen Stellen, dass aufgrund der damals erst geringen Zeitspanne der Rechtsanwendung oder aufgrund von zu wenigen Informationen künftige Beanstandungen nicht auszuschließen seien. ${ }^{122}$ So verwies es im $\mathrm{Zu}-$ sammenhang mit $\$ 1$ Abs. 1 S. 1 Nr. 5 UmwRG darauf, dass sich Art. 9 Abs. 3 AK nicht hauptsächlich auf die Genehmigung von Entwicklungsprojekten beziehe, sondern der dort verwendete Begriff der „Rechtsakte“ weit auszulegen sei, wobei der entscheidende Faktor im eventuellen Verstoß gegen umweltbezogene Vorschriften zu sehen sei. ${ }^{123}$ Sofern $\$ 1$ Abs. 1 S. 1 Nr. 5 UmwRG so gehandhabt werde, dass Maßnahmen wie Emissionsgrenzen für Kfz gericht- lich nicht anfechtbar sind, könnte dies eine Verletzung des Art. 9 Abs. 3 AK darstellen. ${ }^{124}$ Hinsichtlich untergesetzlicher Rechtsvorschriften verwies es auf seine vorherige Stellungnahme zum Inzidentrechtsschutz ${ }^{125}$ und befand aufgrund der zur Verfügung gestellten Informationen und mangels Beweises des Gegenteils, dass derzeit weder ein Verstoß Deutschlands gegen die Entscheidung V/9h noch die Nichteinhaltung des Art. 9 Abs. 2, 3 AK festgestellt werden könne. ${ }^{126}$ Wie diese Einschränkungen zeigen, sind erhebliche Unsicherheiten hinsichtlich des Anwendungsbereichs von Art. 9 Abs. 3 AK zu verzeichnen. Eine Auswertung der Rechtsprechung ergibt verschiedene Möglichkeiten, wie die Verwaltungsgerichte mit diesen umgehen.

Das VG Düsseldorf entschied in Kenntnis der ProtectEntscheidung, dass die Ausklammerung straßenverkehrsrechtlicher Zulassungsentscheidungen von den Umweltrechtsbehelfen aufgrund des Gestaltungsspielraums des Gesetzgebers und der festzulegenden Kriterien für Rechtsbehelfe rechtmäßig sei. Dieser habe den Konflikt zwischen dem völker- und unionsrechtlich gebotenen, möglichst umfassenden Umweltrechtsschutz und dem verfassungsrechtlich garantierten Individualrechtsschutz in Deutschland schonend auflösen dürfen, indem Umweltverbände bei idealtypisch weitreichenden Zulassungsentscheidungen einen weiten Zugang zum Rechtsschutz, nicht jedoch bei Entscheidungen von geringer Umweltrelevanz erhalten. ${ }^{127}$ Trotz der Ausführlichkeit der Entscheidung vermisst man bei ihr eine eingehende Auseinandersetzung damit, dass sich nach dem Vertragstext die von den Mitgliedstaaten festzulegenden, erwähnten Kriterien nur auf die Mitglieder der Offentlichkeit und nicht etwa auf den Anfechtungsgegenstand beziehen, sowie dazu, dass Art. 9 Abs. 3 AK im Unterschied zu Art. 9 Abs. 2 AK für Rechtsakte mit weniger erheblichen Umweltauswirkungen gilt, dafür aber die gerichtliche Überprüfung auf umweltbezogene Vorschriften beschränkt. ${ }^{128}$ Art. 9 Abs. 3 AK stellt allein auf Verstöße gegen umweltbezogene Vorschriften $a b$ und kennt keine einschränkende Erheblichkeitsschwelle.

Nach Meinung des VGH Kassel kann die Ausklammerung der Zielabweichungsentscheidungen nach dem ROG $\mathrm{zu}$ einer Einschränkung des Klagerechts gegen einzelne

\section{3) BT-Drs. 18/9526, S. 36f}

114) ACC, ZUR 2018, 23, 29; dazu, dass für einen ausreichenden Rechtsschutz i.S.d. Art. 9 Abs. 3 AK ggf. eine Ermessensreduzierung bei behördlichen Einschreitungsbefugnissen anzunehmen ist, Schieferdecker, in: Hoppe/Beckmann/Kment, UVPG/ UmwRG, 5. Aufl. 2018, \$1 UmwRG Rdnr. 78.

115) Guckelberger, NuR, 2020, S. 149; Rat der Europäischen Union, Interinstitutionelles Dossier 2018/0089(COD) v. 28.11.2019.

116) BT-Drs. 18/9526, S. 37.

117) Nach OVG Hamburg, Urt. v. 8.4.2019 - 1 Bf 200/15, Rdnr. 130, juris ist kein wesentlicher Unterschied zwischen diesen beiden Maßnahmen erkennbar.

118) Fellenberg/Schiller, in: Landmann/Rohmer, Umweltrecht, 90. EL Juni 2019, UmwRG \$1 Rdnr. 117.

119) OVG Hamburg, Urt. v. 8. 4. 2019-1 Bf 200/15, Rdnr. 130, juris

120) OVG Hamburg, Urt. v. 8. 4.2019-1 Bf 200/15, Rdnr. 134, juris

121) Fellenberg/Schiller, in: Landmann/Rohmer, Umweltrecht, 90. EL Juni 2019, UmwRG \$1 Rdnr. 118.

122) ACC, ZUR 2018, 23, 25.

123) ACC, ZUR 2018, 23, 27.

124) ACC, ZUR 2018, 23, 27; dafür, dass auch Produktgenehmigungen, wie solche für Kfz, Art. 9 Abs. 3 AK unterfallen Dikaios, Überindividueller Umweltrechtsschutz am Beispiel der altruistischen Verbandsklage in der deutschen, griechischen und europäischen Rechtsordnung, 2018, S. 522.

125) ACC, ZUR 2018, 23, 28.

126) ACC, ZUR 2018, 23, 29.

127) VG Düsseldorf, Urt. v. 24.1.2018 - 6 K 12341/17, Rdnr. 224 ff., juris.

128) Zum Konflikt dieser Entscheidung mit der Entscheidung V/9h Sauer, EurUP 2019, 33, 41. 
Festlegungen eines Raumordnungsplans bei bestandskräftig gewordenen Zielabweichungen führen. ${ }^{129}$ Aufgrund der abschließenden Enumeration rechtsbehelfsfähiger Entscheidungen in $₫ 1$ Abs. $1 \mathrm{~S}$. 1 UmwRG und der eindeutigen Gesetzesmaterialien wurden jedoch die Voraussetzungen für eine analoge Rechtsanwendung verneint. ${ }^{130}$ Auch diese Entscheidung ist nicht zufriedenstellend, da sie sich an keiner Stelle mit der Protect-Entscheidung des EuGH auseinandersetzt. Dies könnte ein Hinweis auf die Schwierigkeiten der Rechtsanwendung im Mehrebenensystem sein.

Vereinzelt gewähren die Verwaltungsgerichte trotz der aus ihrer Sicht zu engen Fassung des Anwendungsbereichs dennoch Umweltrechtsschutz. Zu denken ist zum einen an eine angepasste Auslegung des Gesetzeswortlauts oder das Unangewendetlassen der Einschränkung im Hinblick auf die Protect-Rechtsprechung. Am weitesten vorgewagt hat sich das VG Berlin. Weil der Anwendungsbereich des UmwRG aufgrund des Listenprinzips in $\$ 1$ Abs. 1 UmwRG verschlossen war, nach der Protect-Entscheidung aber anerkannte Umweltvereinigungen die Einhaltung objektiv-rechtlicher Umweltschutzvorschriften mit Basis im Unionsrecht gerichtlich überprüfen können lassen müssen, bejahte es eine prokuratorische Klagebefugnis nach $\ 42$ Abs. 2 HS 2 VwGO zur Prüfung der Frage, ob die Verordnung über Ausnahmen von straßenverkehrsrechtlichen Vorschriften für sog. Gigaliner mit dem unionalen Umweltrecht in Einklang steht. ${ }^{131}$

Auch wenn die Auflistung weiterer umweltrechtsbehelfsfähiger Gegenstände in gewissen Bereichen zu einem Mehr an Rechtssicherheit geführt hat, lässt sich - anders als in den Gesetzesmaterialien angenommen wurde - nicht feststellen, dass durch diese Regelungstechnik, ,weiter gehende Abgrenzungsschwierigkeiten und Rechtsunsicherheit" vermieden worden sind. ${ }^{132}$ Angesichts der Unsicherheiten hinsichtlich des sachlichen Anwendungsbereichs des Art. 9 Abs. 3 AK bedarf es weiterer Konkretisierungen auf unionaler oder völkerrechtlicher Ebene zur Klärung der Frage, ob der Katalog des $\$ 1$ Abs. 1 S. 1 UmwRG erweitert werden oder nicht doch zu einer Generalklausel übergegangen werden sollte. Da sich die Gerichtsentscheidungen immer nur auf spezifische Fälle beziehen und schwer prognostizierbar ist, welcher Position das entscheidende Gericht zugeneigt sein wird, stellt sich diese Situation nicht nur für die Rechtsbehelfsführer, sondern auch für diejenigen, deren Vorhaben mit Umweltrechtsbehelfen angegriffen und deren Realisierung dadurch möglicherweise hinausgeschoben wird, als unbefriedigend dar. ${ }^{133}$ Obwohl die Kommission mit ihrer Auslegungsmitteilung zu einem Mehr an Rechtssicherheit beitragen möchte, ${ }^{134}$ ist es letztendlich doch der EuGH, der letztverbindlich über die Auslegung des Unionsrechts zu befinden hat. ${ }^{135}$ Seine Entscheidungen leisten einen bedeutsamen Beitrag dafür, dass sowohl die öffentliche Verwaltung als auch die Rechtspflege effizienter werden können. ${ }^{136}$

Das Vorabentscheidungsverfahren nach Art. 267 AEUV ${ }^{137}$ stellt ein wichtiges Instrument zur Gewährleistung der Kohärenz und Einheitlichkeit der Auslegung des Unionsrechts dar. ${ }^{138}$ Aus diesem Grund sollten die Verwaltungsgerichte vermehrt die Vorlagemöglichkeit nach Art. 267 Abs. 1 AEUV als Instrument zur Feststellung von Auslegungsfragen des Unionsrechts einschließlich des Anwendungsvorrangs gebrauchen. ${ }^{139} \mathrm{Nach}$ den Empfehlungen des EuGH an die nationalen Gerichte bezüglich der Vorlage von Vorabentscheidungsersuchen kann jedes Gericht von dieser Möglichkeit Gebrauch machen, wenn es dies zur Entscheidung eines bei ihm anhängigen Rechtsbehelfs für erforderlich hält. ${ }^{140}$ Angesichts der insoweit dem vorlegenden Gericht zustehenden Einschätzungsprärogative und der Vermutung für die Entscheidungserheblichkeit werden Vorlagen selten an dieser Hürde scheitern. ${ }^{141}$ Bei der Formulierung des Vorabentscheidungsersuchens ist jedoch darauf $\mathrm{zu}$ achten, dass dieses Verfahren nicht auf die Abgabe von Gutachten zu allgemeinen oder hypothetischen Fragen durch den EuGH gerichtet ist. ${ }^{142}$ Der EuGH nimmt in einem solchen Verfahren auch nur zur Auslegung des Unionsrechts Stellung, dessen Anwendung auf den Einzelfall obliegt sodann den nationalen Stellen. ${ }^{43}$ Als besonders nützlich wird ein solches Vorgehen angesehen, wenn es sich um eine neue Auslegungsfrage von allgemeiner Bedeutung für die einheitliche Anwendung des Unionsrechts handelt oder die vorhandene Rechtsprechung auf einen noch nicht vorgekommenen Sachverhalt nicht anwendbar erscheint. ${ }^{144}$ Selbstverständlich greift in Fragen, die ausschließlich nationales Umweltrecht betreffen, das Vorlageverfahren zum EuGH nicht. Dann verbleibt als einziger Weg zur Ausräumung von Unklarheiten die Anrufung des ACC, die auch durch Mitglieder der Öffentlichkeit erfolgen kann. ${ }^{145}$

Es ist daher uneingeschränkt zu begrüßen, dass das VG Schleswig nunmehr in seiner Entscheidung vom 20.11.2019 den EuGH im Wege eines Vorabentscheidungsersuchens die Frage vorgelegt hat, ob es Umweltvereinigungen nach Art. 9 Abs. 3 AK i.V.m. Art. 47 GRCh $^{146}$ möglich sein muss, einen Bescheid gerichtlich anzufechten, mit dem die Produktion von Diesel-Personenkraftwagen mit Abschalteinrichtungen möglicherweise unter Verstoß gegen Art. 5 Abs. 2 Verordnung (EG) Nr. 715/2007 gebilligt wird. ${ }^{147}$

129) VGH Kassel, Beschl. v. 15.8.2019 - 4 B 1303/19, Rdnr. 50, juris

130) VGH Kassel, Beschl. v. 15.8.2019 - 4 B 1303/19, Rdnr. 51 f., juris; ohne Bezug zur Zielabweichung, aber i.E. ebenso OVG Bautzen, Urt. v. 17.8.2018 - 1 A 320/17, Rdnr. 71, juris; VGH München, Beschl. v. 11.4.2018 - 2 CS 18.198, NuR 2019, 483.

131) VG Berlin, Urt. v. 18.4.2018 - 11 K 216.17, ZUR 2018, 497, $498 \mathrm{ff}$; dagegen verneinte das VG Berlin, Urt. v. 31.10.2019$10 \mathrm{~K}$ 412.18, Rdnr. 87, juris bei der auch von Greenpeace unterstützten Klimaklage ein prokuratorisches Recht, weil es für natürliche Personen kein subjektives Recht ausmachen konnte; dazu, dass der Weg über das prokuratorische Recht zur SchlieBung von Rechtsschutzlücken vorzugswürdig ist Bunge, in: ders., UmwRG, 2. Aufl. 2019, \1, Rdnr. 155 f.

132) S. zu diesem Argument für das Listenprinzip BT-Drs. 18/9526, S. 37.

133) Nur bezogen auf die Vorhabenträger Komm., Mitt. v. 28.4.2018, über den Zugang zu Gerichten in Umweltangelegenheiten, C(2017) 2616 final, S. 5.

134) Komm., Mitt. v. 28.4.2018 über den Zugang zu Gerichten in Umweltangelegenheiten, C(2017) 2616 final, S. 5 f.; zu den Wirkungen solcher Mitteilungen Classen, ZHR 2019, 577, $579 \mathrm{f}$

135) S. auch EuGH, Gut. 1/17 v. 30.4.2019, ECLI:EU:C:2019:341, Rdnr. 111 - CETA EU-Kanada.

136) In diese Richtung auch Komm., Mitt. v. 28.4.2018 über den Zugang zu Gerichten in Umweltangelegenheiten, C(2017) 2616 final, S. 5 .

137) Vertrag über die Arbeitsweise der Europäischen Union, AB1 2012 C 326, S. 47.

138) EuGH, Gut. 1/17 v. 30.4.2019, ECLI:EU:C:2019:341, Rdnr. 111 - CETA EU-Kanada.

139) Latzel/Streinz, NJOZ 2013, 97, 98

140) AB1. 2012 I C 338, S. 1, 2.

141) Ehricke, in: Streinz, EUV/AEUV, 3. Aufl. 2018, Art. 267 AEUV Rdnr. 35f.; Karpenstein, in: Das Recht der EU, 67. EL 6/2019, Art. 267 AEUV Rdnr. 25ff.; Wegener, in: Calliess/Ruffert, EUV/AEUV, 5. Aufl. 2016, Art. 267 Rdnr. $22 \mathrm{ff}$

142) EuGH,Urt.v.19. 11.2019-C-585/18u.a.,ECLI:EU:C:2019:982, Rdnr. 70 - A. K.

143) EuGH,Urt.v.19. 11.2019-C-585/18u.a.,ECLI:EU:C:2019:982, Rdnr. 132 - A. K.

144) ABl. 2012 I C 338, S. 2; dazu, dass auf diese Weise eine Präzisierung und Korrektur der EuGH-Rechtsprechung erreicht werden kann, Latzel/Streinz, NJOZ 2013, 97, 98.

145) $\mathrm{Zu}$ dieser Besonderheit des Non-Compliance-Verfahrens Zeitner, EurUP 2019, 159, $163 \mathrm{ff}$.

146) Charta der Grundrechte der Europäischen Union (GRCh), ABl. 2012 C 326, S. 391.

147) VG Schleswig, Beschl. v. 20.11.2019 - 3 A 113/18, BeckRS 2019, 29226. 
Zur Erläuterung legte es dar, dass derartige Entscheidungen nach $\int 1$ Abs. 1 S. 1 Nr. 5 UmwRG nicht umweltrechtsbehelfsfähig sind. Eine analoge Rechtsanwendung hielt es angesichts des Festhaltenwollens des Gesetzgebers am Listenprinzip und der damit fehlenden Planwidrigkeit der Regelungslücke für nicht möglich. Die Annahme eines prokuratorischen Rechts i.S.d. $\$ 42$ Abs. 2 HS 2 VwGO sei abzulehnen, da das Verbot der Verwendung von Abschalteinrichtungen keinen individualschützenden Charakter für natürliche Personen habe. Deshalb solle der EuGH dazu Stellung nehmen, ob sich aus Art. 9 Abs. 3 AK i.V.m. Art. 47 GRCh eine Klagebefugnis unmittelbar aus dem Unionsrecht ergebe. Es sei unklar, ob sich die nach ,,innerstaatlichem Recht festgelegten Kriterien“ nur auf den Kreis der Anfechtungsberechtigten beziehen, oder den Mitgliedstaaten auch Unterscheidungen hinsichtlich der Anfechtungsgegenstände ermögliche, da ,,[a]ufgrund der Vielzahl behördlicher Entscheidungen mit Umweltbezug [...] eine Beschränkung auf bestimmte, im Hinblick auf ihre Umweltauswirkungen schwerwiegende Entscheidungen sinnvoll sein" könnte. ${ }^{148}$ Es bleibt zu hoffen, dass der EuGH demnächst hinsichtlich des deutschen Listenprinzips etwas mehr Licht ins Dunkel bringen wird.

\section{Auffangfunktion der nationalen Gerichte bei Unzulänglichkeiten des Umweltrechtsschutzes gegenüber Unionsmaßnahmen}

Nur der Vollständigkeit halber sei erwähnt, dass das ACC in dem Verfahren ACCC/C/2008/32 part I v. 24. 8.2011 ${ }^{149}$ und part II v. 2. 6. $2017^{150}$ unter Berücksichtigung der Aarhus-Verordnung (EG) Nr. 1367/2006 ${ }^{151}$ und der Auslegung der primärrechtlichen Rechtsschutzregelungen durch den EuGH zu dem Schluss kam, dass die Union selbst die Rechtsschutzvorgaben von Art. 9 Abs. 3, 4 AK unzulänglich umsetzt. In einem Staff Working Dokument der Kommission vom 10.10.2019 werden die bestehenden Umweltrechtsschutzmöglichkeiten analysiert. Rechtsschutzprobleme ergeben sich vor allem daraus, dass die Aarhus-Verordnung einen indirekten Rechtsschutz nur in Bezug auf Maßnahmen des Umweltrechts zur Regelung eines Einzelfalls durch ein Organ oder eine unionale Einrichtung eröffnet, sofern sie rechtsverbindlich sind und Außenwirkung haben (Art. 2 Abs. 1 lit. g), Art. 10 ff. VO Nr. 1367/2006). ${ }^{152}$ Nichtigkeitsklagen von Umweltschutzorganisationen gem. Art. 263 Abs. 4 Var. 2, 3 AEUV scheitern in aller Regel an der engen Auslegung der ,unmittelbaren und individuellen Betroffenheit" durch den EuGH. ${ }^{153}$ Rechtsschutzdefizite seien durch die Mitgliedstaaten zu schließen, die gem. Art. 19 Abs. 1 UAbs. 2 EUV die erforderlichen Rechtsbehelfe vorsehen müssen, um einen wirksamen Rechtsschutz in den vom Unionsrecht erfassten Bereichen zu gewährleisten. Die Praxis in den Mitgliedstaaten variiere, oft fehle es an dem Bewusstsein, dass nationale Rechtsbehelfe zur Schließung von Rechtsschutzlücken direkt gegen Maßnahmen oder Unterlassungen der Union eröffnet sein müssen, um dann über diesen Umweg durch Lancierung eines Vorabentscheidungsersuchens nach Art. 267 AEUV den EuGH zu befassen. ${ }^{154}$

Obwohl Art. 9 Abs. 3 AK - vermittelt über die Auslegung durch den EuGH - erhebliche Auswirkungen für den Rechtsschutz gegenüber Maßnahmen oder Unterlassungen nationaler Behörden zeitigt, ist nach den Aussagen im Arbeitsdokument kaum davon auszugehen, dass die Union Anpassungen beim bestehenden primärrechtlichen Rechtsschutz vornehmen wird. So werden der Ausgestaltungsspielraum der Vertragsparteien beim Rechtsschutz nach Art. 9 Abs. 3 AK $^{155}$ ebenso wie der Umstand betont, dass ein internationales Abkommen nicht die Machtverteilung nach den Verträgen ändern könne, insbesondere die Autonomie der Unionsrechtsordnung gewahrt werden müsse. ${ }^{156}$ Dies deckt sich mit den Ausführungen des
EuGH zum CETA-Abkommen, wonach außerhalb des Gerichtssystems der Union stehende Spruchkörper keine Zuständigkeit für die Auslegung oder Anwendung des Unionsrechts haben und ihre Entscheidungen nicht dazu führen dürfen, ,dass die Unionsorgane daran gehindert werden, gemäß dem verfassungsrechtlichen Rahmen der Union zu funktionieren“. ${ }^{157}$ Wenn somit den nationalen Gerichten zur Implementierung der Rechtsschutzanforderungen des Art. 9 Abs. 3 AK gegenüber Maßnahmen oder Unterlassungen der Union eine Auffangfunktion zukommt, wird sich langfristig die Frage nach einer Erweiterung des Kreises der umweltrechtsbehelfsfähigen $\mathrm{Ge}-$ genstände stellen, um bestehende Rechtsschutzlücken auf Unionsebene zu schließen. Da sich die für das Rechtsverhältnis i.S.v. $\$ 43$ Abs. 1 VwGO maßgeblichen Normen auch aus dem Unionsrecht ergeben können ${ }^{158}$ und ein Rechtsverhältnis bereits dann besteht, wenn ,die Anwendung einer bestimmten Norm des öffentlichen Rechts auf einen bereits überschaubaren Sachverhalt streitig ist," 159 liegt eine Feststellungsklage $(\mathbb{S} 43 \mathrm{VwGO})$ nahe, ${ }^{160}$ etwa mit dem Inhalt, das Nichtbestehen eines Rechtsverhält-

148) VG Schleswig, Beschl. v. 20.11.2019 - 3 A 113/18, BeckRS 2019, 29226 Rdnr. $41 \mathrm{f}$.

149) Report of the Compliance Committee - Findings and recommendations with regard to communication ACCC/C/2008/32 (Part I) concerning compliance by the European Union v. 14. 4.2011, Stand 19.2.2020, abrufbar unter https://www.unece.org/fileadmin/DAM/env/pp/compliance/CC-32/ece. mp.pp.c.1.2011.4.add.1_as_submitted.pdf.

150) Report of the Compliance Committee - Findings and recommendations with regard to communication ACCC/C/2008/32 (Part II) concerning compliance by the European Union v. 17.3.2017, Stand 19.2.2020, abrufbar unter https://www.unece.org/fileadmin/DAM/env/pp/compliance/CC-57/ece. mp.pp.c.1.2017.7.e.pdf; zu den Besonderheiten dieses Verfahrens Zeitner, EurUP 2019, 159, $166 \mathrm{ff}$.

151) Verordnung (EG) Nr. 1367/2006 des Europäischen Parlaments und des Rates vom 6.9.2006 über die Anwendung der Bestimmungen des Übereinkommens von Århus über den Zugang zu Informationen, die Öffentlichkeitsbeteiligung an Entscheidungsverfahren und den Zugang zu Gerichten in Umweltangelegenheiten auf Organe und Einrichtungen der Gemeinschaft, ABl. 2006 L 264, S. 13.

152) Komm., Staff working Document v. 10.10.2019, SWD(2019) 378 final, S. 9, 11, $21 \mathrm{ff}$

153) Komm., Staff working Document v. 10.10.2019, SWD(2019) 378 final, S. 12 ff.; nur am Rande sei erwähnt, dass auch Individualnichtigkeitsklagen an diesem Erfordernis scheitern, da Einzelpersonen in der Regel keine individualisierten Adressaten einer Umweltmaßnahme im Sinne der Plaumann-Formel sind, s. auch UfU, Toolkit, unter Nr. 4.1.3, Stand 19.2.2020, abrufbar unter https://www.ufu.de/wp-content/uploads/2016/ 10/Zugang-zu-Gerichten-in-Umweltangelegenheiten-inDeutschland-Toolkit.pdf.

154) Komm., Staff working Document v. 10.10.2019, SWD(2019) 378 final, S. 16.

155) Komm., Staff working Document v. 10.10.2019, SWD(2019) 378 final, S. 6.

156) Komm., Staff working Document v. 10.10.2019, SWD(2019) 378 final, S. 4.

157) EuGH, Gut. 1/17 v. 30.4.2019, ECLI:EU:C:2019:341, Rdnr. 118 - CETA EU-Kanada.

158) Guckelberger, in: FS für Wendt, S. 1165; Giegerich/Lauer, ZEuS 2014, 461, 474; Lenz/Staeglich, NVwZ 2004, 1421, 1426.

159) BVerwG, Urt. v. 30.11.2011 - 6 C 20.10, BVerwGE 141, 223, 225.

160) So auch Sobotta, Rechtsschutz gegen EU-Maßnahmen, Vortrag bei der Fachtagung Wissenschaftliche Unterstützung des Rechtsschutzes in Umweltangelegenheiten in der 19. Legislaturperiode am 2.12.2019, Folie 21, Stand 19.2.2020, Folien abrufbar unter https://www.ufu.de/wp-content/uploads/2016/ 10/sobotta_praesentation_dt.pdf; ohne Bezug zur AK, aber bezogen auf unionale Rechtsschutzdefizite Guckelberger, in: FS für Wendt, S. 1165, 1177. 
nisses zwischen einer Person und einer Kontrollbehörde wegen der Ungültigkeit der Unionsregelung, aus der sich die Kontrollfunktion der Behörde ergibt, festzustellen. In Konstellationen, in denen sich eine solche nationale Kontrollbehörde nicht identifizieren lässt, bleibt die Bestimmung eines Klagegegners auf nationaler Ebene bei eigentlich unionsrechtlichen Umweltmaßnahmen schwierig und ist daher eine Schaffung von Rechtsschutzmöglichkeiten auf Unionsebene eindeutig die vorzugswürdige Alternative.

\section{Fazit}

Zwanzig Jahre nach Inkrafttreten der AK besteht weiterhin Unklarheit über die Reichweite der Rechtsbehelfe zur Erfüllung der Anforderungen des Art. 9 Abs. 3 AK. Viel spricht dafür, dass Deutschland trotz der Erweiterung der Liste der rechtsbehelfsfähigen Gegenstände seinen völkerund unionsrechtlichen Verpflichtungen noch nicht in ausreichendem Maße nachgekommen ist. Dass zusätzlich die nationalen Gerichte ggf. einen Umweltrechtsschutz gegen Handlungen oder Unterlassungen der Organe bzw. Einrichtungen der Union eröffnen müssen, weil die Union ohne solche Auffangrechtsbehelfe ihren eigenen Verpflich- tungen nach Art. 9 Abs. 3 AK unzulänglich nachkommen würde, ist angesichts der weitreichenden Einwirkungen von Art. 9 Abs. 3 AK auf die Ausgestaltung des nationalen Rechtschutzes irritierend.

Open Access. Dieser Artikel wird unter der Creative Commons Namensnennung 4.0 International Lizenz veröffentlicht, welche die Nutzung, Vervielfältigung, Bearbeitung, Verbreitung und Wiedergabe in jeglichem Medium und Format erlaubt, sofern Sie den/die ursprünglichen Autor(en) und die Quelle ordnungsgemäß nennen, einen Link zur Creative Commons Lizenz beifügen und angeben, ob Änderungen vorgenommen wurden.

Die in diesem Artikel enthaltenen Bilder und sonstiges Drittmaterial unterliegen ebenfalls der genannten Creative Commons Lizenz, sofern sich aus der Abbildungslegende nichts anderes ergibt. Sofern das betreffende Material nicht unter der genannten Creative Commons Lizenz steht und die betreffende Handlung nicht nach gesetzlichen Vorschriften erlaubt ist, ist für die oben aufgeführten Weiterverwendungen des Materials die Einwilligung des jeweiligen Rechteinhabers einzuholen.

Weitere Details zur Lizenz entnehmen Sie bitte der Lizenzinformation auf http://creativecommons.org/licenses/by/4.0/deed.de.

Open access funding provided by Projekt DEAL.

\section{Denkmalschutz als Anwendung umweltbezogener Rechtsvorschriften*}

\section{Jörg Spennemann}

(c) Springer-Verlag GmbH Deutschland, ein Teil von Springer Nature 2020

\begin{abstract}
Die Neufassung des Umwelt-Rechtsbehelfsgesetzes (UmwRG) ${ }^{1}$ vom 29. 5. 2017 hat neben Regelungen zur Fehlerbehebung eine Erweiterung seines Anwendungsbereichs durch eine Ausweitung des Katalogs der rechtsbehelfsfähigen Gegenstände gebracht; u. a. sind nun auch Klagen gegen Einzelgenehmigungen für Vorhaben, die keiner Pflicht zur Umweltprüfung unterliegen, möglich. Dies wirft die Frage auf, welche Auswirkungen sich daraus für Genehmigungsentscheidungen der Bauaufsichts- und Denkmalschutzbehörden ergeben. Neben der neuen Anfechtungsmöglichkeit von Einzelgenehmigungen ist Dreh- und Angelpunkt der dem UmwRG zugrunde liegende Umweltbegriff. Sollte dieser den Denkmalschutz in vollem Umfang umfassen, wären landesgesetzliche Regelungen zur Einführung von Klagebefugnissen von Vereinigungen insoweit unzulässig.
\end{abstract}

\section{Rechtlicher Rahmen}

\subsection{Gegenstände des Denkmalschutzes; Bezüge zum Bundesrecht}

Das Denkmalrecht schützt Baudenkmäler (als einzelne oder als Mehrheit von baulichen Anlagen²), Gründenkmäler (z.B. Park- und Gartenanlagen), bewegliche Denkmäler, Boden- oder archäologische Denkmäler sowie - je nach landesrechtlicher Ausgestaltung - Kulturlandschaften. ${ }^{3}$ Hinzu kommt ein landesrechtlich unterschiedlich ausgestalteter Schutz der Denkmäler vor Auswirkungen von Veränderungen in ihrer Umgebung. ${ }^{4}$ Nahezu alle Verän-

Dr. Jörg Spennemann, Oberlandesanwalt,

Landesanwaltschaft Bayern,

München, Deutschland derungen werden einer Verfahrenspflicht unterworfen; die Genehmigung kann erteilt werden, wenn eine beabsichtigte Maßnahme denkmalverträglich oder aus überwiegen-

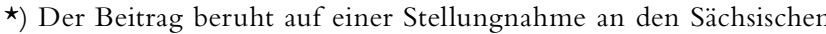
Landtag zum Entwurf eines Gesetzes zur Einführung von Mitwirkungsrechten und zum Verbandsklagerecht für anerkannte Denkmalschutzvereinigungen vom 13.9. 2018 (LT-Drs.6/14736); er gibt die private Rechtsauffassung des Verfassers wieder.

1) Gesetz über ergänzende Vorschriften zu Rechtsbehelfen in Umweltangelegenheiten nach der EG-Richtlinie 2003/35/EG (Umwelt-Rechtsbehelfsgesetz) v. 23.8.2017, BGBl. I S. 3290; zuletzt geändert durch Art. 4 des Gesetzes v. 17.12.2018, BGBl. I S. 2549.

2) Bei Mehrheiten von baulichen Anlagen kann es sich je nach landesrechtlicher Lesart auch um Ensembles, Denkmalzonen oder Denkmalbereiche handeln.

3) Vgl. zu den Baudenkmälern Davydov, in: Martin/Krautzberger, Handbuch Denkmalschutz und Denkmalpflege, 4. Aufl. 2017, Teil C, Rdnr. $1 \mathrm{ff}$;; zu den Bodendenkmälern Kemper, in: Martin/Krautzberger, Handbuch Denkmalschutz und Denkmalpflege, 4. Aufl. 2017, Teil C, Rdnr. 102 ff.; zu den Gründenkmälern Hönes, in: Martin/Krautzberger, Handbuch Denkmalschutz und Denkmalpflege, 4. Aufl. 2017,Teil C, Rdnr. $134 \mathrm{ff}$; zu den Sachgesamtheiten; Martin, in: ders/Krautzberger, Handbuch Denkmalschutz und Denkmalpflege, 4. Aufl. 2017, Teil C, Rdnr. 144ff.; zu den Kulturlandschaften Dornbusch, in: Martin/Krautzberger, Handbuch Denkmalschutz und Denkmalpflege, 4. Aufl. 2017, Teil C, Rdnr. $187 \mathrm{ff}$.

4) Davydov, in: Martin/Krautzberger, Handbuch Denkmalschutz und Denkmalpflege, 4. Aufl. 2017, Teil C, Rdnr. 174 ff.; zu den Schutzobjekten insgesamt s. auch Oebbecke, in: Ehlers/Fehling/Pünder, Besonderes Verwaltungsrecht, Bd. 2, 3. Aufl. 2013, \$42 Rdnr. $13 \mathrm{ff}$ 